\title{
Flora und Vegetation der Dörfer im nordöstlichen Burgenland
}

\author{
von UWE RAABE, Borgholzhausen, und DIETMAR BRANDES, Braunschweig
}

mit 1 Figur und 17 Tabellen

\begin{abstract}
The object of this paper was to investigate and to describe the flora and the vegetation of villages in north-eastern Burgenland (Austria). On the basis of floristical mapping of 14 villages it is possible to give statements about the frequency of plants typical for villages. The high constancy of many species is remarkable; 88 species were to be found in at least half of the villages. The village flora of NE Burgenland is characterized by numerous continental and/or thermophilous species, whereby the part of neophytic plants is relatively high.

Comparisons of village floras of other regions show - in relation to Central Europe that there exist only a few "typical village plants". The village flora is rather different from landscape to landscape, caused especially by climatic but also edaphic and cultural-historical reasons.

At the borders of the villages ruderal vegetation is developed on an extraordinary scale. 25 ruderal plant communities are documented by plant sociological relevées. Characteristic communities for the investigation area are: A t riplice t u $\mathrm{m}$ a $t$ a $\mathrm{r}$ i a e, basal com-

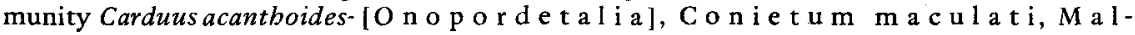
ve tum pusilla e, On opordet um a canthi, Panicum capillare community, Salvio-M a r ubietum peregrini and Sclerochloo-Polygonet $\mathbf{u} \mathbf{m}$ a $\mathbf{v}$ i cula $\mathrm{r}$ is. The ruderal shrubberies are built up by Lycium barbarum, Sambucus nigra, Ailantbus altissima and Robinia pseudacacia.
\end{abstract}

\section{Inhaltsverzeichnis}

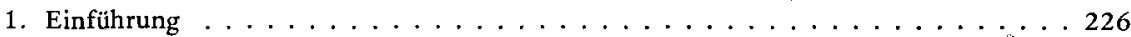

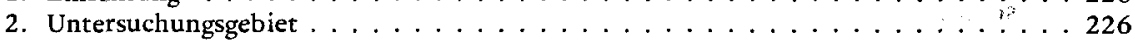

3. Flora der Dörfer . . . . . . . . . . . . . . . . . . . . . . 228

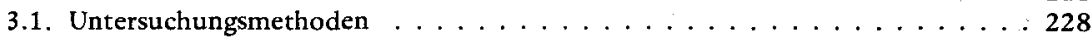

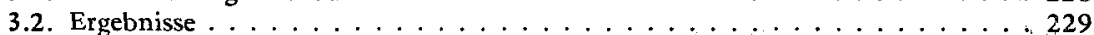

3.3. Vergleich der Dorffloren verschiedener Gebiete Mitteleuropas . . . . . . . 232

3.4. Allgemeine Gedanken zur Dorfflora . . . . . . . . . . . . . . . . 234

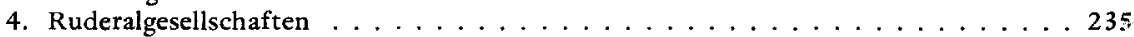

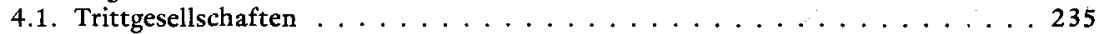

4.1.1. Sclerochloo-Polygonetum avicularis (Gams 1927)

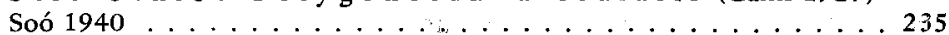

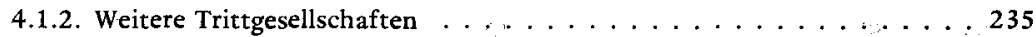

4.2. Einjährige Gänsefuß-Gesellschaften ( $\mathrm{C}$ h e n o p o d i e t e a $\mathrm{Br}$-Bl.

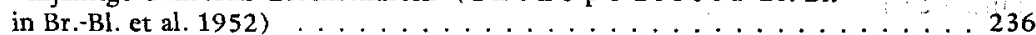

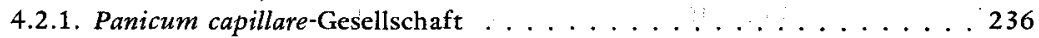

4.2.2. Horde e $\mathrm{t} \mathrm{um} \mathrm{murini} \mathrm{Libb.1933} \mathrm{.} \mathrm{.} \mathrm{.} \mathrm{.} \mathrm{.} \mathrm{.} \mathrm{.} \mathrm{.} \mathrm{.} \mathrm{.} \mathrm{.} 237$

4.2.3. Cha enarrhino-Chenopodiet um botyros:

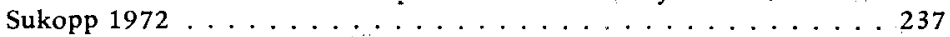

4.2.4. Chenopodietum vulvaria e Gutte et Pyšek 1976.... 237

4.2.5 Atriplicet um tataricae Ubrizsy $1949 \ldots \ldots 238$ 
4.2.6 Malvet um p us ill a e Morariu 1943 ............. 239

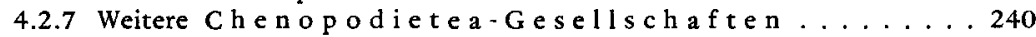

4.3. Wärmebedürftige Distel-Gesellschaften ( $\mathrm{n}$ op or d i o $\mathrm{n}$ a $\mathrm{c}$ a $\mathrm{th}$ i i

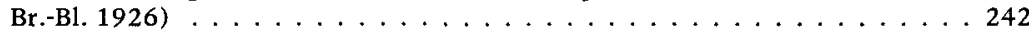

4.3.1. On op ordet um a canth i i Br.-Bl. (1923 n.n.) 1926...... 242

4.3.2. Marrubium vulgare-Gesellschaft . . . . . . . . . . . . . . . 244

4.3.3. Basalgesellschaft Carduus acanthoides- [O n o p or d e $t$ a $l$ i a $] \ldots 244$

4.3.4. S a l vio-M a r r b i e t um peregrin i Mucina $1981 \ldots 244$

4.3.5. Salvia nemorosa-Straßenrandgesellschaft . . . . . . . . . . . 248

4.3.6. Potentillo argenteae-Artemisietum absinthi

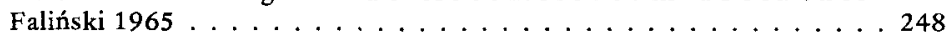

4.4. Klettenfluren (A r ction la p p a e Tx. $1937 \mathrm{em} .1950$ ).........252

4.4.1. A r c t i o-Arte m is i e t u m (Tx. 1942) Oberd. ap. Oberd.

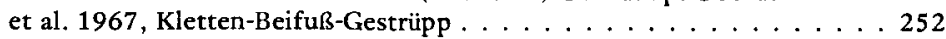

4.4.2. Con i t $\mathrm{um} \mathrm{ma} \mathrm{culat} \mathrm{i}$ Pop (1965) $1968 \ldots \ldots . \ldots 252$

4.4.3. S a m bu ce $t$ u $m$ e b u li Felf. $1942 \ldots \ldots \ldots \ldots$

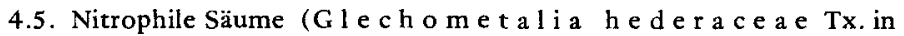

Tx. et Brun-Hool 1975) . . . . . . . . . . . . . . . . . . . 254

4.6. Ruderale Gebüsche . . . . . . . . . . . . . . . . . . . . 255

4.6.1. Lycium barbarum-Bestände . . . . . . . . . . . . . . . . . . 255

4.6.2. Ailantbus altissima-Robinia pseudacacia-Gehölze . . . . . . . . 255

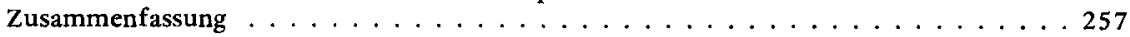

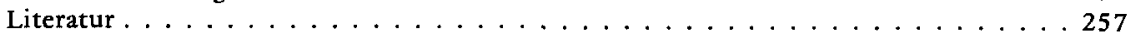

\section{Einführung}

Der Lebensraum Dorf fand erst relativ spät das Interesse der Geobotanik. Auf der Grundlage weniger Arbeiten erfolgten schnell Verallgemeinerungen, die unseres Erachtens für größere Gebiete kaum gültig sein können. Viele Untersuchungen erfolgten zudem in Gebieten mit bereits stark verarmter Dorfflora.

Um einen soliden Überblick über die (mittel-)europäische Dorfvegetation zu bekommen, ist es notwendig, in möglichst verschiedenen Wuchsräumen und Landschaften jeweils eine größere Anzahl von Dörfern sowohl floristisch als auch pflanzensoziologisch zu untersuchen.

Wir legen hiermit unsere Ergebnisse aus dem nordöstlichen Burgenland vor, das, am südöstlichen Rande Mitteleuropas gelegen, auch heute noch eine außerordentlich reizvolle Dorfflora aufweist.

\section{Untersuchungsgebiet}

Die untersuchten Dörfer liegen in einer Meereshöhe von ca. 117-200 m im sogenannten Seewinkel sowie auf der angrenzenden Parndorfer Platte und gehören damit bereits zur Kleinen Ungarischen Tiefebene. Das Klima zeigt deutlich subkontinentale Tönung mit hohen Juli-Mitteln (Neusiedl a.S.: $20,1^{\circ} \mathrm{C}$ ) und relativ geringen Jahresniederschlägen (Neusiedl a.S.: $609 \mathrm{~mm}$ ). Das Klima wird stark vom Neusiedler See beeinflußt, der stärkere Temperaturextreme ausgleicht. Mit einem Jahresmittel von fast $10^{\circ} \mathrm{C}$ gehört das nördliche Burgenland zu den wärmsten Gebieten Österreichs (KLETTER 1976). 


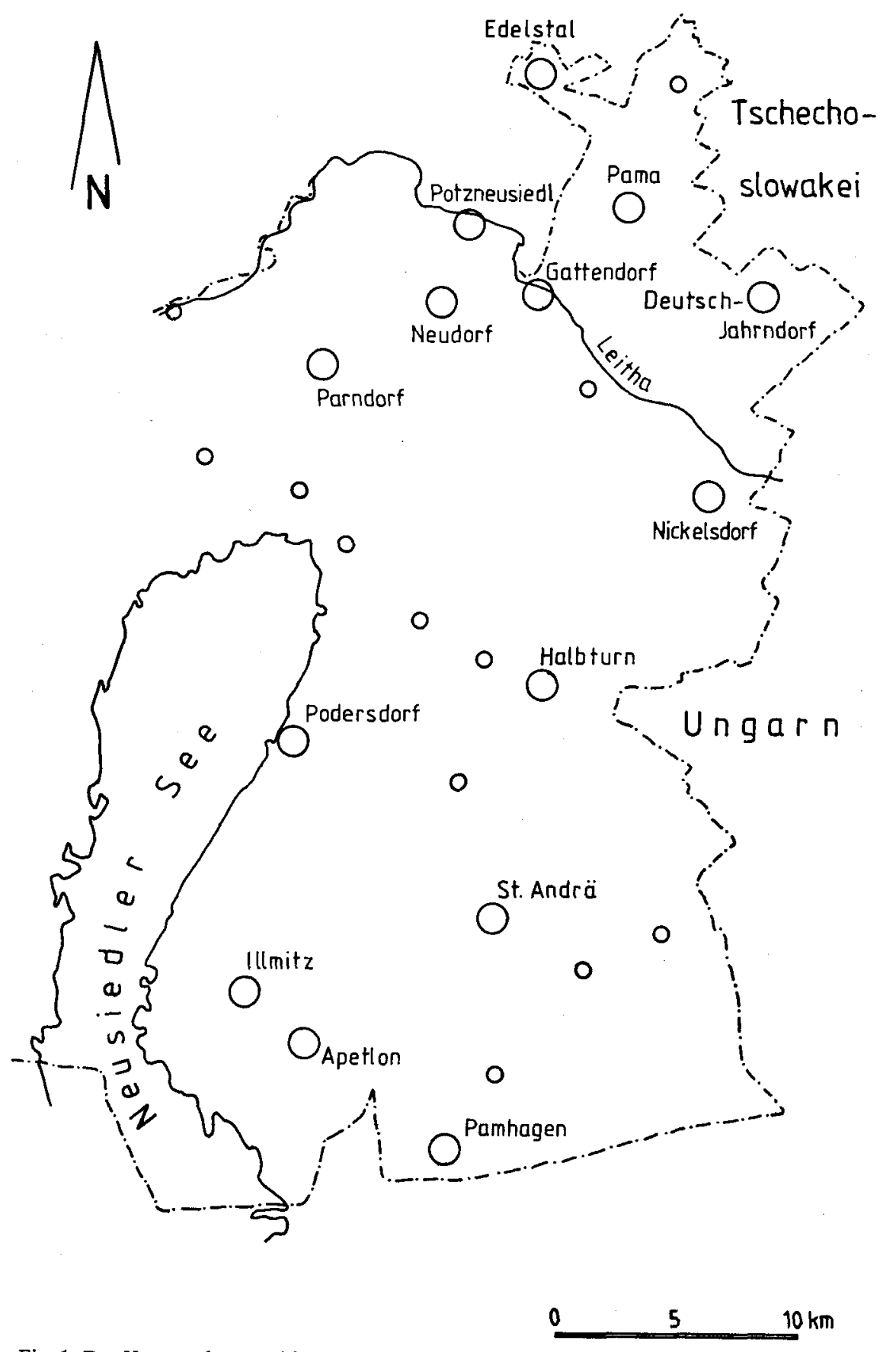

Fig. 1. Das Untersuchungsgebiet. 
Die natürliche Vegetation des Gebietes wurde weitestgehend von Eichenmischwäldern gebildet, lediglich auf Sonderstandorten hat es kleinflächig edaphisch bedingte Steppen gegeben. Die Wälder wurden bis auf kleine Reste zurückgedrängt. Selbst von den einst ausgedehnten Hutweiden ist nur wenig geblieben. Heute prägen Acker und Rebgärten das Bild der Landschaft, im Seewinkel finden sich verschiedene Sonderkulturen (z.B. Paprika, Tomaten, Kürbis, Hirse und Sonnenblumen).

Etwa zwischen dem 11. und 13. Jahrhundert entstanden im nordöstlichen Österreich planmäßig angelegte Angerdörfer. Die Dorfmitte, der grüne Anger, war Gemeindebesitz (Allmende) und diente als gemeinsamer Wirtschafts- und Festort (STENZEL 1984). Viele Siedlungen verödeten im Verlaufe der Türkenkriege, so daß nach 1700 weitausgedehnte Ingenieurdörfer begründet werden mußten (KRÄFTNER 1984). Während die Schmalseite der Streckhöfe auf den Anger blickt, wurden die Wirtschaftsgebäude wegen der Feuersgefahr vom Ortskern abgerückt und zu ,Scheunengassen“ zusammengefaßt. Auf diese Weise entstanden für Mitteleuropa außerordentlich großflächige Dörfer.

Im Bereich des Angers (,Hauptstraße“, ,Hauptplatz“) ist auch in den untersuchten Dörfern nur noch wenig Platz für spontane Vegetation. Ein großer Teil der Flächen wurde asphaltiert oder gepflastert, mit Bäumen und Ziersträuchern bepflanzt, mit dekorativen Rabatten versehen, zu Vorgärten umgewandelt. Die verbliebenen Gras- bzw. Rasenflächen werden \pm regelmäßig gemäht.

Floristisch und vegetationskundlich wesentlich interessanter sind in der Regel die Seitengassen, die Höfe sowie vor allem die Gassen hinter den Höfen, an denen sich Scheunen und die für den Seewinkel so charakteristischen hölzernen Maisspeicher (Tschardaken) aufreihen. Dort sind die Wege häufig noch nicht asphaltiert; an Wegrändern, auf Schutthaufen sowie am Rande von Sambucus nigraGebüschen bzw. Robinia pseudacacia-Gehölzen finden sich Ruderalgesellschaften in einem für Mitteleuropa ungewöhnlichen Ausmaß.

Bislang sind die Dörfer des Untersuchungsgebietes von der sog. ,,Verstädterung" wesentlich weniger betroffen als die anderer Gebiete Mitteleuropas. Allerdings hat auch hier - mit einer gewissen Verzögerung - ein kaum noch aufzuhaltender Wandel eingesetzt. Von der zunehmenden Erschließung für den Tourismus sind die Orte des Seewinkels besonders betroffen: So sind die für die Dörfer früher so charakteristischen Gänsescharen längst aus dem Straßenbild verschwunden, selbst Hühner sieht man immer seltener in den Gassen. Auch im nordöstlichen Burgenland werden seit einigen Jahren Wege, Straßen und Plätze zunehmend versiegelt, Freiräume werden gärtnerisch gestaltet und intensiv gepflegt. Dorfteiche sind bereits die Ausnahme; sind sie noch vorhanden, werden sie z.B. als Angelgewässer genutzt wie in Apetlon.

\section{Flora der Dörfer}

\subsection{Untersuchungsmethoden}

In insgesamt 14 Dörfern des nordöstlichen Burgenlandes (vgl. Fig. 1) wurde eine Auswahl \pm dorftypischer Pflanzenarten kartiert. Als Grundlage für diese Kartie- 
rung diente ein für Nordrhein-Westfalen zusammengestellter Erhebungsbogen (LIENENBECKER 1986), der aber um zahlreiche Arten ergänzt werden mußte, die in Nordrhein-Westfalen fehlen oder nur sehr selten auftreten.

Um diese Arten möglichst vollständig zu erfassen, wurden in den Dörfern jeweils alle öffentlich zugänglichen Plätze, Gassen und Brachflächen abgesucht. Soweit möglich, wurden auch die Höfe und Gärten mit in die Untersuchung einbezogen. Unberücksichtigt blieben lediglich Bahnanlagen, da sich ihre Flora meist recht deutlich von der eigentlichen dörflichen Flora unterscheidet.

Alle Dörfer wurden mindestens zweimal aufgesucht, im Juni 1985 bzw. im Juni 1986 und im September 1985. Sicherlich wurde dabei das eine oder andere Vorkommen übersehen, im wesentlichen dürften die in Tabelle 1 aufgeführten Arten aber vollständig erfaßt sein.

\subsection{Ergebnisse}

In Tabelle 1 sind 129 (ohne Mentha spec. und Verbascum spec.) \pm dorftypische Pflanzenarten aufgeführt, die in den untersuchten Dörfern nachgewiesen werden konnten. Einige kritische Sippen, die im zeitigen Frühjahr erscheinen und bereits im Juni nur noch ausnahmsweise angetroffen wurden, Solidago-Arten sowie einige Arten der Gattungen Amarantbus, Atriplex und Sisymbrium wurden weggelassen.

Von den in der Tabelle 1 aufgeführten Arten (incl. Mentha spec. und Verbascum spec.) wurden durchschnittlich 86,5 Sippen je Dorf notiert, wobei die höchste Zahl (94) in Parndorf, die niedrigste (70) in Podersdorf erreicht wurde. Folgendermaßen verteilen sich die Arten (ohne Mentba spec. und Verbascum spec.) auf die fünf Stetigkeitsklassen:

$\begin{array}{ll}\text { I } & \text { (in } 1-20 \% \text { der Dörfer): } 19 \text { Arten } \\ \text { II } & \text { (in } 21-40 \% \text { der Dörfer): } 15 \text { Arten } \\ \text { III } & \text { (in } 41-60 \% \text { der Dörfer): } 21 \text { Arten } \\ \text { IV } & \text { (in } 61-80 \% \text { der Dörfer): } 13 \text { Arten } \\ \text { V } & \text { (in } 81-100 \% \text { der Dörfer): } 61 \text { Arten }\end{array}$

Auffällig groß ist die Anzahl der Sippen, die in mindestens $81 \%$ der untersuchten Dörfer angetroffen wurde; dies ist fast die Hälfte der aufgeführten Arten. 88 Arten wurden in mindestens der Hälfte der Ortschaften notiert.

Die hohe Stetigkeit so vieler Arten dürfte vor allem auf das recht homogene Untersuchungsgebiet und die einheitliche Struktur der Orte zurückzuführen sein, aber auch darauf, daß die Veränderungen der Dörfer in diesem Raum bei weitem noch nicht so stark fortgeschritten sind wie in anderen mitteleuropäischen Landschaften.

Dennoch lassen sich durchaus gewisse Unterschiede in der Zusammensetzung der Flora des nördlichen und des südlichen Gebietsteils erkennen. So haben Aegopodium podagraria, Aetbusa cynapium, Alliaria petiolata, Lamium maculatum, Parietaria officinalis und Sagina procumbens ihren Schwerpunkt im nördlichen Teil des Untersuchungsgebietes, während z.B. Ambrosia artemisiifolia, Melilotus alba, Panicum capillare (ohne Bahnhofs-Vorkommen!), Puccinellia 
Tabelle 1. Vorkommen ,typischer" Dorfpflanzen in Dörfern des nordöstlichen Burgenlandes.

Aegopodium podagraria Aethusa cynapium Alliaria petiolata

3 * Althaea of ficinalis Amaranthus albus A. retroflexus Ambrosia artemisiifolia

3 Anthriscus caucalis

A. cerefolium

Arctium lappa

A. minus

A. tomentosum

Arenaria serpyllifolia

Aristolochia clemat it is

Armoracia rusticana

Artemisia absinthium

A. annua

A. vulgaris

Asperugo procumbens

Asplenium ruta-muraria

A. trichomanes

Atriplex hastata agg.

A. nitens

A. patula

1 A. rosea

A. tatarica

Ballota niqra

Bromus sterilis

B. tectorum

Bryonia alba

B. dioica

Carduus acanthoides

C. nutans

Chaenarrhium minus

Chelidonium majus

Chenopodium album

c. bonus-henricus

C. botrys

C. glaucum

c. murale

c. hybridum

C. polyspermum

$2 / 3$ C. rubrum agg.

3 C. urbicum

C. vulvaria

Conium maculatum

Conyza canadensis

Coronopus squamatus

Cymbalaria muralis
Cynoglossum officinal

Datura stramonium

Descurainia sophia

Diplotaxis tenuifolia

Dipsacus fullonum

Echium vulgare

Eragrostis poaeoides

Euphorbia lathyris

E. peplus

Fallopia dumetorum

Gelinsoga ciliata

G. parviflora

Geranium pyrenaicum

Hesperis matronalis

Hordeum mur inum

Hyoscyamus niger

Impatiens parviflora

Kochia scoparia
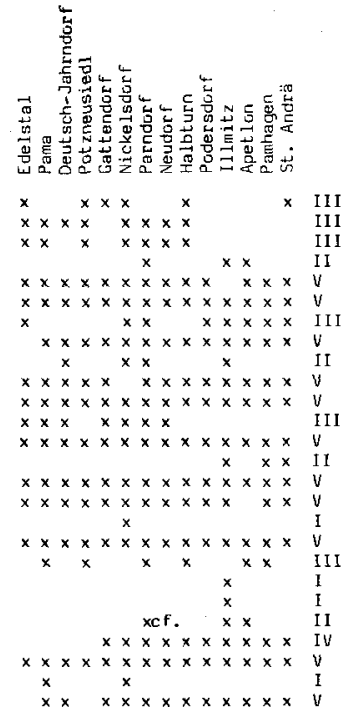

$\times \times \times \times \times \times \times \times \times \times \times \times \times \times$ $x \times \times \times \times \times \times \times \times \times \times \times \times \times \times \quad \times$

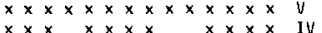
$\times \times \times \times \times \times \times \times \times \times \times \times$ IV

$\times \times \times \times \times \times \times \times \times \times \times \times \times \times$

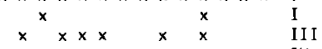

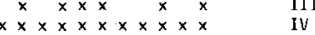

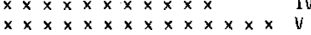

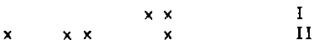

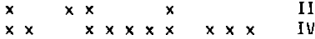

$x \times \times \quad x \times \times x \times x \times x \times y$

$x \times \times \times \times \times \times \times \times \times \times \times \times$

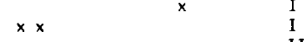

$\times \times \times \times \times \times \times \quad x \quad$ III

$\times \times \times \times \times \times x \times x \times x \times x y$

$\times \times \times \times \times \times \times \times \times \times \times \times \times \times y$

$x \times x \times$ II

$x \times \times \times \times \times \times \times \times \times \times \times \times \times$ V $\times \times \times \times \times \times \times \times \times \times \times \times \times \times 4$

$\times \times \times \times \times \times \times \times \times \times \times \times \times \times 4$

$\times \times \times \times \times \times \times \times \times \times \times \times \times \times \quad$

$\times \times \times \times \times \times \times \times \times \times \times \times V$

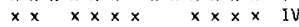

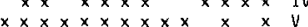

$\times \times \times \times \times \times \times \times \times \times \times \times \quad V$

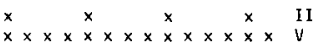

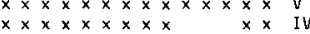

$x \quad x \quad x \quad x \quad x \quad 11$

$x \times \times \times \times x \times x \times x \times x y$

$\times \times \times \times \times \times \times \times \times \times \times \times \times \quad y$

$x \times \times \times \times \times \times \times \times \times \times \times$

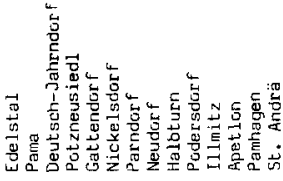

Lactuca serriola

Lamium maculatum

3 Lappula squarrosa

Lavathera thuringiaca

Leonurus cardiaca

2 L. marrubiastrum

Lepidium campestre

$L$. densiflorum

L. ruderale

Linaria vulgaris

Malva neglecta

M. pusilla

$M$. sylvestris

Marrubium peregrinum

M. vulgare

Matricaria discoidea

Melilatus albus

Mentha spec.

Mentha $x$ villosa

Myosoton aquaticum

Nepeta cataria

Oenothera biennis agg.

Onopordon acanthium

Oxalis corniculata

o. europaea

Panicum capillare

Papaver somniferum

Parietaria officinalis

Phytolacca acinosa

Poa compressa

Portulaca oleracea

Potentilla anserina

$P$. argentea

P. reptans

$P$. supina

Puccinellia distans

Reseda lutea

R. luteola

Reynoutria japonica

Rumex patientia

Sagina procumbens

Salsola kali

Sambucus ebulus

Sambucus nigra

Saponaria officinalis

Sclerochloa dura

Sedum acre

Setaria verticillata

Silene noctiflora

Silybum marianum

5. irio

5. loeselii

S. officinale

Solanum nigrum

Stenact is annua

Tanacetum balsamita

T. parthenium

T. vulgare

Urt ica dioica

u. urens

Verbascum spec. ${ }^{* *}$

Verbena officinal is

Viola odorata $x \times \times \times \times \times \times \times \times \times \times \times \times V$

$x \times x^{x} \times x \times{ }^{x}$ II

$\times \times \times \times \times \times \times \times \times \times \times \times \times \times \quad$

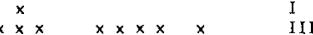

$\begin{array}{llllll}x & x & x & x & x & \text { II }\end{array}$

$x \times x \times x \times x \times x \times x x x x$

$\times \times \times \times \times \times \times \times \times \times \times \times \times \times \quad$

$x \times \times \times \times \times \times \times \quad \times \times \times \times \quad$

$x \times x \quad x \times \quad x \quad x$ III

$\begin{array}{lllllllll}x \times & \times & \times & \times & \times & \times & \times \times \times & \times & V \\ x \times & \times & \times & \times & \times & \times & \times & \text { III }\end{array}$

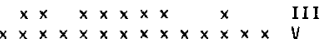

$x \times x \times x \times x \quad x$ II

$x \times \times \times \times \times \times \times \times \times \times$

$x \times \times \times \times \times \times \times \times \times \times \times \times \times \frac{1}{x}$

$x \quad x \quad$ II

$x \times \times \times \quad x \times \quad x \quad$ III

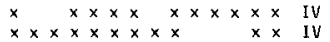

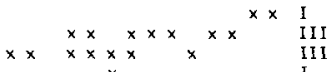

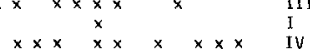

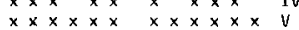

$\times \times \times \times \times \times \times \times \times \times \times \times \times \times \quad$

$\times \times \times \times \times \times \times \times \quad \times \times \times \times$.

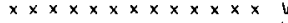

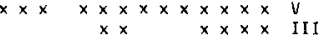

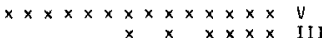

$\times \quad \times \times \times \times \times \times \times \times \times \times \times \quad V$

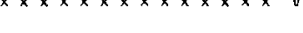

$x \times x \quad x \times \quad \times \times \times$ III
$\times \times \times \times x$ III

$x \times x \quad x \times$ III

$\times \times \times \times \times \times \times \times \times \times \times \times \times \times$.

$\times \times \times \times \times \times \times \times \times \times \times \times \times V$

$x \times \times \times \times \times \times \times \quad \times \times \times \times \times$
$x \times \times$

$\begin{array}{llllllllllllll}x & x & x & x & x & x & x & x & x & x & I V\end{array}$

$\times \times \times \times \times \times \times \times \times \times \times \times \times V$

$x \times \times \times \times \times \times \times x \quad$ IV

$x \times \times \times \times \times \times \times \times \times \times \times$ I

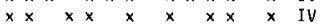

$x \times x \times x \times x \times x \times x \times x \times y$

$x \times x \times x \times x \times x \times x \times x \times y$

$\times \times \times \times \times \times \times \times \times \times \times \times \times$
$\times \times \times$

$x \times x \times x \quad x \times x \quad$ III

$\times \times \times \times \times \times \times \times \times \times \times \times \times \times 4$

$\times \times \times \times \times \times \times \times \times \times \times \times \times V$

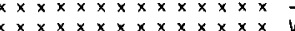

$\times \times \times \times \times \times \times \times \times \times \times \times x \times$

* Rote Liste Usterreich (Niklfeld et al, 1986)

* Div. Gartenminzen, excl. M. x villosa

$\checkmark$. thapsus, $v$. densiflorum et phlomoides 
distans und Reseda luteola im südlichen Teil häufiger angetroffen wurden. Ausschlaggebend sind hierfür vor allem die naturräumlichen Gegebenheiten; so finden Arten frischerer Standorte in den Dörfern des nördlichen Teils - drei dieser Orte sind unmittelbar am Rande der Leithaaue aufgereiht - eher geeignete Wuchsorte als in dem trockeneren und vielleicht auch noch stärker wärmebegünstigten Seewinkel. Chenopodium vulvaria, Marrubium vulgare und Nepeta cataria sind im Seewinkel nur noch selten anzutreffen, während sie sich im nördlichen Teil des Untersuchungsgebietes noch in den meisten Ortschaften gehalten haben. Die unterschiedliche Häufigkeit dieser empfindlichen Arten ist möglicherweise in der stärkeren Veränderung der Dörfer des Seewinkels begründet ${ }^{1}$.

Der Rückgang der Dorfflora ist im nordöstlichen Burgenland wesentlich geringer als in Teilen der Bundesrepublik Deutschland, was sich auch in den entsprechenden ,Roten Listen" deutlich widerspiegelt. Marrubium vulgare gilt jedoch bereits auch in Österreich als vom Aussterben bedroht, Chenopodium vulvaria als stark gefährdet (NIKLFELD et al. 1986). Neben diesen beiden Arten sind 14 weitere der in Tabelle 1 aufgeführten Arten in Österreich in unterschiedlichem Ausmaße gefährdet.

Sippen wie Asplenium ruta-muraria, Asplenium trichomanes und Cbenopodium bonus-benricus dürften mangels geeigneter Standorte im Untersuchungsgebiet immer selten gewesen sein.

Als Charakteristikum der dörflichen Flora wird in der Literatur öfter angeführt, daß der Anteil an Neophyten im Vergleich zu Städten gering und der Anteil an Archäophyten besonders hoch sei (FALIŃSKI 1971, SUKOPP 1983). Tabelle 1 zeigt jedoch deutlich, daß der Neophytenanteil an der Dorfflora des Untersuchungsgebietes relativ hoch ist, was sicher nicht auf eine „Verstädterung" der Ortschaften zurückgeführt werden kann. Die Ursache dürfte vielmehr im günstigen Klima des pannonischen Raumes liegen, bekanntlich wird die heutige Pflanzendecke Ungarns weitgehend von Neophyten geprägt.

Auf die einzelnen Arten soll aus Platzgründen im folgenden nicht weiter eingegangen werden. Bezüglich ihrer Verbreitung im östlichen Österreich sei auf JANCHEN (1977) verwiesen. Die von JANCHEN für Apetlon und Illmitz angegebene Atriplex rosea konnte von uns leider dort ebensowenig mehr bestätigt werden wie Leonurus marrubiastrum für Apetlon.

Neben den in Tabelle 1 aufgeführten Arten wurden einige weitere, größtenteils nicht dorftypische Sippen notiert, deren Fundorte aufgrund der Seltenheit bzw. Gefährdung hier mitgeteilt werden sollen:

Centrantbus ruber (Parndorf), Cyperus fuscus (Apetlon), Cyperus pannonicus (Apetlon), Hibiscus trionum (Apetlon), Lytbrum byssopifolia (Neudorf), Silene dicbotoma (Halbturn).

Die beiden Cyperus-Arten sind nach NIKLFELD et al. (1986) gefährdet, Hibiscus trionum und Lytbrum byssopifolia stark gefährdet.

\footnotetext{
1 Chenopodium vulvaria findet sich allerdings auch in Städten des östlichen Österreichs (Eisenstadt, Wien: Schwarzenbergplatz!).
} 


\subsection{Vergleich der Dorffloren verschiedener Gebiete Mitteleuropas}

In Tabelle 2 wird die Stetigkeit von $50 \pm$ dorftypischen Pflanzenarten in Dörfern unterschiedlicher Gebiete Mitteleuropas verglichen. Zur Verfügung standen dabei neben den Ergebnissen aus dem nordöstlichen Burgenland Daten aus dem Ostbraunschweigischen Flachland, dem Ostbraunschweigischen Hügelland, dem Westfälischen Tiefland ${ }^{2}$, dem westlichen Münsterland, dem Osnabrücker Hügelland, dem Lippischen Bergland und dem Sauerland. Die Angaben für die fünf letztgenannten Räume gehen auf Bestandsaufnahmen im Rahmen des Kartierungsprojektes der Arbeitsgemeinschaft für biologisch-ökologische Landesforschung (ABÖL), Münster, zurück ${ }^{3}$.

Nur ein verhältnismäßig kleiner Teil der in Tabelle 2 aufgeführten Arten ist den Dörfern aller Gebiete gemeinsam. Viele Arten sind sehr unterschiedlich verbreitet, wobei sich in erster Linie die geographische Lage der untersuchten Ortschaften bemerkbar macht. Das Klima des Burgenlandes ist am stärksten kontinental getönt; eine Reihe von thermophilen und/oder kontinentalen Arten wie Atriplex tatarica und Kocbia scoparia ist auf diesen Raum beschränkt. Andererseits fehlen dort Arten, die in den stärker atlantisch geprägten Gebieten regelmäßig in den Dörfern auftreten, wie z. B. Lamium album ${ }^{4}$. Verstärkt werden diese Unterschiede noch durch die klimatisch begünstigte Lage der planar-kollinen Stufe gegenüber der des Berglandes. Wärmeliebende bzw. trockenheitsertragende Arten treten verstärkt im Tiefland auf, sind im Bergland deutlich seltener oder fehlen ganz. Dies zeigt sich besonders im Sauerland, auch wenn bei der Betrachtung der Dörfer dieses Raumes zu berücksichtigen ist, daß die Ortschaften eines relativ uneinheitlichen Raumes und auch unterschiedlicher Höhenlagen zusammengefaßt werden mußten, weil die Zahl der kartierten Dörfer für eine Untergliederung noch nicht ausreichend erschien. Arten, die im Sauerland fehlen bzw. deutlich zurücktreten, sind z.B. Ballota nigra s. I., Solanum nigrum und Galinsoga parviflora. Andererseits wurden aber auch hier einige an das Berg- und Hügelland gebundene Arten notiert. Eine Besonderheit der Flora der Dörfer des Sauerlandes stellt das präalpine Peucedanum ostrutbium (OBERDORFER 1983) dar, das zudem weitestgehend auf die höheren Lagen des Sauerlandes begrenzt ist.

Die Stetigkeitsangaben der Tabelle 2 spiegeln jeweils die aktuellen Verhältnisse wider. Einige Arten kamen früher auch in Gebieten vor, in denen sie heute - zumindest in Dörfern - vergeblich gesucht werden oder aber sie sind deutlich seltener geworden. So fand sich Marrubium vulgare früher auch im Westfälischen Tiefland und im Osnabrücker Hügelland (KOCH 1958), Cbenopodium bonusbenricus war gerade im Berg- und Hügelland viel häufiger. Besonders betroffen

\footnotetext{
${ }^{2}$ Dörfer im nordwestlichen Kreis Minden-Lübbecke und angrenzenden Niedersachsen im Bereich des Mindener Flachlandes und der Diepholzer Moorniederung.

${ }^{3}$ Die Zusammenstellung zur Dorfflora von Nordrhein-Westfalen von WITTIG \& RÜCKERT (1985) ist u.a. aufgrund einer anderen Kartierungsmethode nicht vergleichbar. Ebenso sind auch die Arbeiten von HILBRICH et al. (1983) sowie von EICHSTAD' (1986) nur bedingt vergleichbar.
}

41987 konnte Lamium album in einem sehr kleinen Bestand in Mönchhof gefunden werden. 
Tabelle 2. Stetigkeit ausgewählter Dorfpflanzen in verschiedenen Naturräumen Mitteleuropas.

$\begin{array}{rrrrrrrrrr} & \text { NÖB } & \text { OBF } & \text { OBH } & \text { WFT } & \text { WM } & \text { OSH } & \text { LB } & S & \\ \text { Zahl der untersuchten Dörfer } & 14 & 9 & 11 & 23 & 80 & 40 & 68 & 91 & \text { ges. } 336\end{array}$

Nur im Burgenland

$\begin{array}{lr}\text { Atriplex tatarica } & V \\ \text { Kochia scoparia } & V \\ \text { Marrubium peregrinum } & V \\ \text { Rumex patientia } & V \\ \text { Sclerochloa dura } & V \\ \text { Sisymbrium loeselii } & V \\ \text { Carduus acanthoides } & V \\ \text { Chenopodium vilvaria } & \text { III } \\ \text { Marrubium vulgare } & \text { III }\end{array}$

Deutlicher Schwerpunkt im Osten bzw. im Tiefland

Amaranthus retroflexus

Chenopodium murale

Onopordon acanthium

Lepidium ruderale

Mercurialis annua

Descurainia sophia

Chenopodium hybridum

Hordeum murinum

Datura stramonium

Ieonurus cardiaca

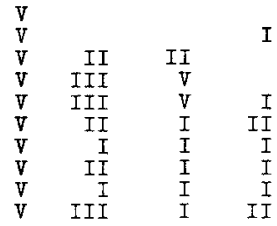

Im Sauerland fehlend bzw. deutlich zurücktretend

Ballota nigra agg.
Solanum nigrum
Galinsoga parviflora
Bromus sterilis
Malva neglecta
Arctium minus
Urtica urens
Oxalis europaea

$\begin{array}{rrr}V & V & V \\ V & I I & I \\ V & I V & I I I \\ V & V & V \\ V & V & V \\ V & I I & I V \\ V & V & I V \\ I V & I I I & I I\end{array}$

IV
$\mathrm{V}$
$\mathrm{V}$
$\mathrm{IV}$
$\mathrm{V}$
$\mathrm{V}$
$\mathrm{V}$
$\mathrm{V}$

\begin{tabular}{|c|c|c|}
\hline I & $I$ & I \\
\hline IV & III & II \\
\hline IV & IV & I \\
\hline II & IV & II \\
\hline II & III & II \\
\hline III & V & II \\
\hline IV & IV & III \\
\hline IV & III & IV \\
\hline
\end{tabular}

Im Burgenland fehlend bzw. deutlich zuricktretend
Sagina procumbens
Chaerophyllum temulum
I II
$\operatorname{IIT}$
$\begin{array}{rrrr}V & \text { IV } & V & V \\ \text { II } & V & I I I & I \\ V & V & V & V\end{array}$
Schwerpunkt im subatlantischen Gebirge bzw. Higelland
Chenopodium bonus-henricus
Polypodium vulgare
Cystopteris fragilis
Myrrhis odorata
Peucedanum ostruthium
II $\quad I$

$\begin{array}{rrr}I & I I & \text { III } \\ I V & I I & I \\ I I I & I I I & I I \\ I & I & I\end{array}$

In den Dörfern aller untersuchten Gebiete verbreitet

\begin{abstract}
Matricaria discoidea
Sisymbrium officinale

Aegopodium podagraria

Chelidonium majus

Euphorbia peplus

Potentilla anserina
\end{abstract}

\section{Sonstige}

Armoracia rusticana

Artemisia absinthium

Asplenium ruta-muraria

Bryonia dioica

Conium maculatum

Galinsoga ciliata

Hesperis matronalis

Tanacetum vulgare

Verbena officinalis
I

$\begin{array}{cl}I & I \\ I & I\end{array}$

NOB $=$ nordöstl. Burgenland

OBF = Ostbraunschw. Flachland

$\mathrm{OBF}=$ Ostbraunschw. Flachland
$\mathrm{OBH}$

WFT $=$ Westf. Tiefland (s. Text!)

$W M=$ westliches Munsterland

$\mathrm{OSH}=$ Osnabricker Hügel land

$\mathrm{LB}=\mathrm{Lipper}$ Bergland

$\mathrm{S}=$ Sauerland

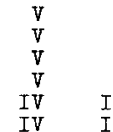

$\begin{array}{rr}V & \\ V & \\ V & \\ V & \\ I V & I V \\ I V & \text { III }\end{array}$

$\begin{array}{rrr}V & V & V \\ V & V & I I I \\ V & V & V \\ V & V & I I I \\ I V & I V & I V \\ I V & V & I I I\end{array}$

\begin{tabular}{|c|c|c|c|c|c|c|c|}
\hline$V$ & IV & IV & II & I & II & IV & III \\
\hline $\mathrm{V}$ & & & IV & I & $\mathrm{I}$ & $I$ & I \\
\hline I & V & IV & $I$ & I & V & III & I \\
\hline III & & & & $I$ & $I$ & & \\
\hline $\mathrm{V}$ & I & & III & I & II & I & I \\
\hline II & II & $I$ & V & $\mathrm{V}$ & IV & IV & II \\
\hline I I & I & II & I & I & I & II & III \\
\hline III & IV & $I$ & V & V & $\mathrm{V}$ & III & III \\
\hline V & II & II & $I$ & & II & I & \\
\hline
\end{tabular}

$$
\begin{aligned}
I & =\text { in } 1-20 \% \\
I I & =\text { in } 21-40 \% \\
I I I & =\text { in } 41-60 \% \\
I V & =\text { in } 61-80 \% \\
V & =\text { in } 81-100 \% \text { der Dörfer }
\end{aligned}
$$


von dieser Verarmung der Dorfflora ist das westliche Münsterland, wo heute selbst Malva neglecta eine ausgesprochene Seltenheit ist. Andererseits sind z.B. Amarantbus retroflexus im westlichen Münsterland oder Lepidium ruderale im Sauerland keine typisch dörflichen Pflanzenarten, sondern meist (vorübergehende) Einschleppungen der jüngsten Zeit. Oft werden diese Arten nur im Bereich von Bahnanlagen angetroffen.

\subsection{Allgemeine Gedanken zur Dorfflora}

Aus den bisher vorliegenden Bestandsaufnahmen zur Dorfflora lassen sich einige allgemeine Aussagen ableiten (vgl. auch WITTIG \& RUCKERT 1985, DECHENT et al. 1986):

- Die Flora läßt sich im Hinblick auf ihr Vorkommen in Dörfern in verschiedene Gruppen gliedern, wobei den grundsätzlich nicht in Dörfern vorkommenden Sippen die ausschließlich in Dörfern vorkommenden Arten gegenüberstehen. Dazwischen gibt es allerdings fließende Übergänge, außerdem kann das Verhalten einzelner Sippen in verschiedenen Naturräumen völlig unterschiedlich sein.

- Artenreichtum und -zusammensetzung sind zunächst von den natürlichen standörtlichen Voraussetzungen abhängig, d.h. von der Struktur der Dörfer, von ihrer geographischen Lage, von den naturräumlichen Gegebenheiten sowie in zunehmendem Maße vom Nutzungs- und Funktionswandel (,Verstädterung“").

- Viele Arten sind in den Dörfern auf ganz bestimmte Strukturelemente angewiesen (z.B. Mauerpflanzen oder Arten der Dorfteiche). Diese Arten können natürlich für entsprechende Strukturen in Städten gleichermaßen typisch sein. Auch hier gibt es wieder gleitende Übergänge.

- Die Bezeichnung bestimmter Arten als ,Verstädterungszeiger" ist nur mit einiger Vorsicht möglich und kann häufig nur für einzelne Gebiete gelten.

- „Dorfpflanzen“" können durchaus auch in Städten auftreten, soweit entsprechende Strukturen vorhanden sind. Ihr Anteil an der Gesamt-Vegetation einer Stadt ist im allgemeinen jedoch gering. Eine klare Trennung zwischen ,typisch dörflicher" und ,,typisch städtischer" Flora ist also nur bedingt möglich.

- Die Einheitlichkeit der Flora der Dörfer eines bestimmten Raumes - und damit die Zahl hochsteter Arten - ist von mehreren Faktoren abhängig. Die Flora ist zunächst um so einheitlicher, je einheitlicher Struktur und Größe der Dörfer sind. Mit den zunehmenden Veränderungen der Dörfer in neuerer Zeit ist einerseits der Rückgang bestimmter Sippen verbunden, andererseits treten Arten neu auf; die Zahl der hochsteten Arten nimmt jedoch ab. Ein ,intakter" Zustand der dörflichen Flora eines bestimmten Raumes ist somit nicht unbedingt mit der Zahl der insgesamt festgestellten Arten verknüpft, sondern wird eher vom Anteil hochstet vorkommender Sippen indiziert.

- Es gibt nur relativ wenige für ganz Mitteleuropa typische Dorfpflanzen. Die dörfliche Flora ist vielmehr von Landschaft zu Landschaft verschieden, vor allem aus klimatischen, aber auch aus edaphischen und kulturhistorischen Gegebenheiten.

Versuche zur Wiedereinbürgerung ,,typischer Dorfplanzen" müssen daher von der gebietstypischen Dorfflora ausgehen. Jedes pauschale Vorgehen würde nur zu einer Florenverfälschung führen. 


\section{Ruderalgesellschaften}

\subsection{Trittgesellschaften}

\subsubsection{Sclerochloo-Polygonetum avicularis (Gams 1927) Soó 1940} (Tab. 3)

Das Sclerochloo-Polygone tum avicularis ist die bezeichnende Trittgesellschaft mitteleuropäischer Trockengebiete. So wurde es bislang aus dem Wallis, dem nördlichen Oberrhein-Hügelland, Unter- und Mittelfranken, aus Thüringen, Südmähren und der Südslowakei beschrieben. Die kleinflächigen, in der Regel aber recht homogenen Bestände werden von Sclerocbloa dura, Polygonum aviculare agg., Poa annua und Lepidium ruderale aufgebaut. Auf das auffällige Zurücktreten von Plantago major wies schon OBERDORFER (1983a) hin.

Tabelle 3. Sclerochloo-Polygonetum avicularis (Gams 1927) Soó 1940.

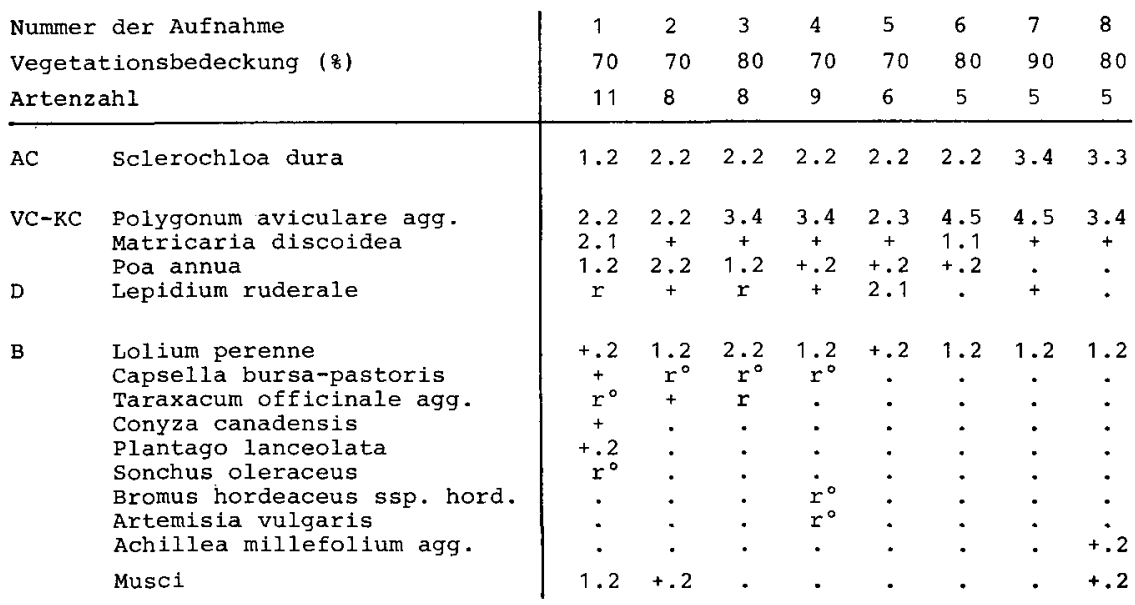

\subsubsection{Weitere Trittgesellschaften}

Auf sandigen und \pm voll besonnten Wegen sind durch Eragrostis minor, Polygonum aviculare agg., Digitaria sanguinalis und Portulaca oleracea gekennzeichnete Trittgesellschaften verbreitet, die vermutlich alle zum $\mathrm{P}$ ol y g o n e $\mathrm{t} \mathrm{u} \mathrm{m} \mathrm{c}$ a lc a t i Lohm. 1975 gehören.

Auf den großen Angern findet man die Lolium perenne-Plantago major-Gesellschaft ebenso wie auf beschatteten und nur wenig befahrenen Kellergassen.

Einzelaufnahme 1:

Hauptplatz von Pama. $10 \mathrm{~m}^{2}$, D $100 \%$. 13.8.1985:

VC-KC: 3.3 Lolium perenne, 1.1 Plantago major, 3.3 Potentilla anserina, 2.2 Trifolium repens, 2.2 Achillea millefolium agg., 1.2 Plantago lanceolata, 1.2 Trifolium pratense, 1.1 Taraxacum officinale agg., + Dactylis glomerata; B: 2.2 Medicago lupulina, 1.2 Convolvulus arvensis. 
In den Fahrspuren der Kellergassen wird die ausdauernde Lolium perennePlantago major-Gesellschaft vom $\mathrm{P}$ o $1 \mathrm{y}$ g o $\mathrm{no}-\mathrm{M}$ a $\mathrm{t} \mathrm{r}$ i c a r i e t u m d is c o i d e a e (Siss. 1969) Tx. 1972, der Zentralassoziation des Verbandes $M$ a tricario-Polygonion avicularis (Br.-Bl. 1931) Riv. Mart. 1975, ersetzt.

Einzelaufnahme 2:

Edelstal. $2 \mathrm{~m}^{2}$, D $70 \%$. 13.8.1985:

AC: 2.3 Matricaria discoidea; VC: 4.3 Polygonum aviculare agg.; B: 1.1 Plantago major, + Lolium perenne.

\subsection{Einjährige Gänsefuß-Gesellschaften (Chenopodietea Br.-Bl. in Br.-Bl. et al. 1952)}

\subsubsection{Panicum capillare-Gesellschaft (Tab. 4)}

$\mathrm{Zu}$ den besonders auffälligen Pflanzengesellschaften des Untersuchungsgebietes zählt die Panicum capillare-Gesellschaft. Dichte Bestände der Haar-Hirse säumen das Gleisbett der Eisenbahnstrecke Neusiedl-Pamhagen vor allem in den Ortsrandbereichen in einer Breite von $20-200 \mathrm{~cm}$. Neben dem dominanten Panicum capillare erreichen lediglich Conyza canadensis, Amarantbus retroflexus, Setaria viridis und Lolium perenne höhere Stetigkeit (vgl. Tab. 4). Diese artenarme Gesellschaft steht sowohl der Ordnung $\mathrm{S}$ i s y $\mathrm{m}$ b ri e t a $1 \mathrm{i}$ a als auch dem Verband $\mathrm{E}$ r a g r o s t i o n nahe. In wenigen Fällen wurde Panicum capillare auch am Rande von Maisfeldern in größeren Beständen beobachtet.

Tabelle 4. Panicum capillare-Gesellschaft.

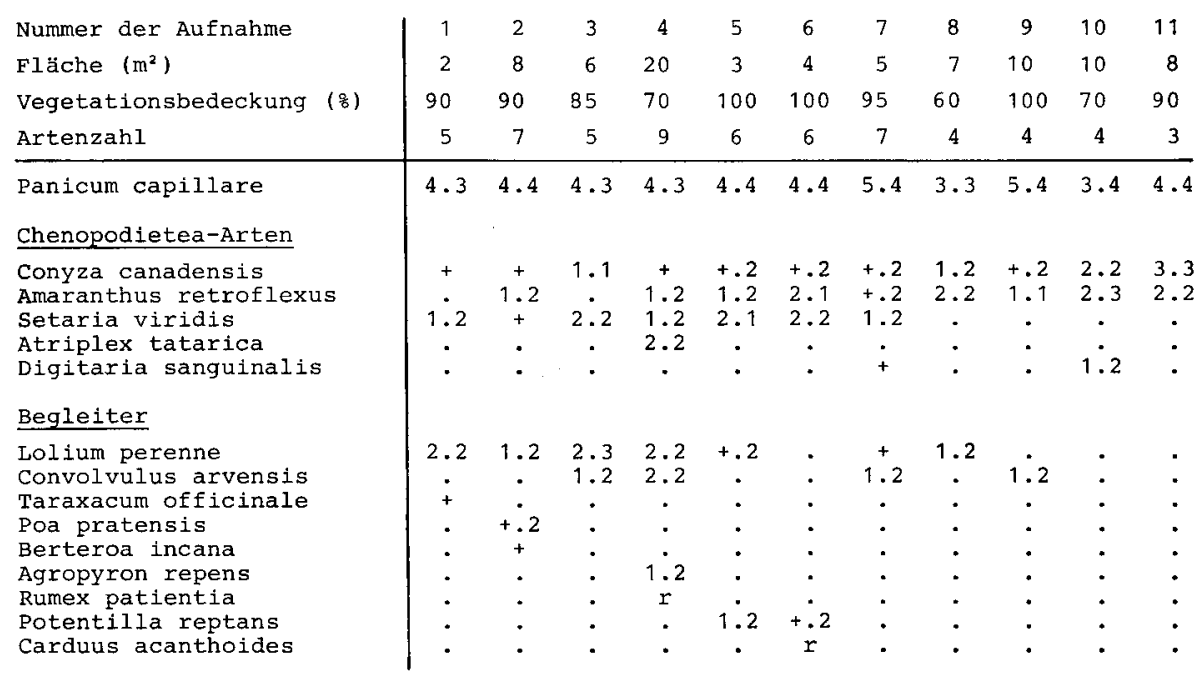


Panicum capillare ist ein Neophyt nordamerikanischer Herkunft, der in seiner Heimat offene bzw. gestörte Böden, sandige Prärien, Straßenränder und Flußufer besiedelt (SCHABERG 1983). In Europa dehnte sich dieses dekorative Gras vermutlich von Gärten und Friedhöfen aus.

Die Panicum capillare-Bestände sind offensichtlich relativ neu, werden sie doch weder von JANCHEN (1977) noch von FORSTNER (1984) für das Burgenland erwähnt. Inzwischen sind auch aus dem benachbarten Stadtrandgebiet von Bratislava entsprechende Bestände bekannt geworden (FERÁKOVÁ \& JAROLÍMEK 1987).

\subsubsection{Hordeetum murini Libb. 1933}

Wenn Hordeum murinum in den Dörfern des nördlichen Burgenlands auch recht verbreitet ist, so scheinen Bestände des $\mathrm{H}$ or de e $\mathrm{t} \mathrm{m} \mathrm{m} \mathrm{u} \mathrm{ri} \mathrm{n} \mathrm{i}$ doch selten zu sein.

Einzelaufnahme 3:

Purbach, Mauerfuß einer Kellergasse. $4 \mathrm{~m}^{2}$, D $95 \%$. 14.8.1985:

AC: 3.3 Hordeum murinum; D: 3.3 Malva sylvestris; KC: + Amarantbus retroflexus; B: 1.2 Lolium perenne, 2.2 Polygonum aviculare agg., 1.1 Artemisia vulgaris, + Carduus acantboides.

\subsubsection{Chaenarrhino-Chenopodietum botyros Sukopp 1972}

Auf planiertem Kies fand sich vor dem Schloß Halbturn das $\mathrm{Ch}$ a e $\mathrm{n}$ a $\mathrm{r} \mathbf{r} \mathrm{h} \mathrm{i}$ $\mathrm{n}$ o- Che nop od i e $\mathrm{t} \mathrm{m}$ bot y r os mit zahlreichen floristisch interessanten Arten als schüttere und niedrigwüchsige Pioniergesellschaft.

Einzelaufnahme 4:

$70 \mathrm{~m}^{2}, \mathrm{D} \cdot 5 \%$. 15.8.1985:

AC: 1.1 Cbenopodium botrys; DA: + Chaenarrbinum minus; VC: + Salsola kali; OC, KC: 1.1 Setaria viridis, 1.1 Cbenopodium album, + Mercuralis annua, $\mathrm{r}$ Amarantbus retroflexus, $\mathrm{r}$ Amarantbus albus, r Setaria glauca; B: + Ajuga charnaepitys, + Eragrostis minor,+ Lappula squarrosa,$+{ }^{\circ}$ Polygonum lapatbifolium, r Ecbinocbloa crus-galli, r Fallopia convolvulus.

\subsubsection{Chenopodietum vulvariae Gutte \& Pyšek 1976}

Zumindest aus der Bundeshauptstadt Eisenstadt kann auch das $\mathrm{C}$ h e $\mathrm{n}$ o p od i e $\mathrm{t} \mathrm{u} \mathrm{m} \mathrm{v} \mathrm{u} \mathrm{l} \mathrm{vari} \mathrm{a} \mathrm{e} \mathrm{mit} \mathrm{einer} \mathrm{Aufnahme} \mathrm{belegt} \mathrm{werden.} \mathrm{Vermutlich}$ kommt es auch noch in einigen Dörfern des Untersuchungsgebietes vor.

Einzelaufnahme 5:

Eisenstadt, Mauer des Schloßparks. 2 x 0,2 $\mathrm{m}^{2}$, D $15 \%$. 14.8.1985:

AC: 2.2 Cbenopodium vulvaria; KC: + Sonchus oleraceus; B: 1.1 Poa annua,+ Polygonum aviculare agg. , + Oxalis corniculata. 


\subsubsection{Atriplicetum tataricae Ubrizsy 1949 (Tab. 5)}

Das A t r i p l i c e $t$ u $m$ t a $t$ a $r$ i c a ist an den Dorfrändern, entlang der Scheunengassen und auf Müllplätzen häufig anzutreffen. Charakterart dieser wärmeliebenden und trockenheitsertragenden Assoziation ist Atriplex tatarica, die im allgemeinen bestandsbildend auftritt. Die Zugehörigkeit zum $\mathrm{S}$ i s y $\mathrm{m} \mathrm{b} \mathrm{r}$ i o $\mathrm{n}$ wird durch die Anwesenheit von Hordeum murinum, Cbenopodium strictum, Conyza canadensis u.a. dokumentiert. Örtlich läßt sich neben einer Ausbildung von Hordeum murinum eine solche von Kocbia scoparia unterscheiden (Tab. 5).

Vom A $t r$ i 1 i c e $t$ u $m$ t $t$ a $r$ i a e sind bislang Aufnahmen aus Ungarn, der CSSR und der DDR (mitteldeutsches Trockengebiet) bekannt geworden; eine Übersichtstabelle findet sich bei BRANDES (1982).

Tabelle 5. Atriplicetum tataricae Ubrizsy 1949.

\begin{tabular}{|c|c|c|c|c|c|c|c|c|c|c|}
\hline Nummer der Aufnahme & 1 & 2 & 3 & 4 & 5 & 6 & 7 & 8 & 9 & 10 \\
\hline Fläche $\left(\mathrm{m}^{2}\right)$ & 3 & 2 & 7 & 3 & 6 & 6 & 4 & 5 & 15 & 40 \\
\hline Vegetationsbedeckung $(8)$ & 95 & 100 & 90 & 90 & 90 & 90 & 90 & 98 & 90 & 70 \\
\hline Artenzahl & 8 & 7 & 6 & 6 & 10 & 9 & 6 & 10 & 13 & 13 \\
\hline Atriplex tatarica & $4 \cdot 3$ & 4.4 & 5.4 & 4.4 & 4.4 & 4.4 & $2 \cdot 3$ & $2 \cdot 3$ & 3.3 & 4.4 \\
\hline $\begin{array}{l}a_{1} \text { Hordeum marinum } \\
\text { Lolium perenne }\end{array}$ & $\begin{array}{l}3.2 \\
1.2\end{array}$ & 1.2 & $\begin{array}{c}2.2 \\
+ \\
\end{array}$ & 1.2 & $\begin{array}{c}2.2 \\
. \\
\end{array}$ & $\begin{array}{l}+.2 \\
1.2 \\
\end{array}$ & $\begin{array}{l}2 \cdot 2 \\
1.1 \\
\end{array}$ & $\dot{\bullet}$ & $\dot{\bullet}$ & $\dot{\bullet}$ \\
\hline 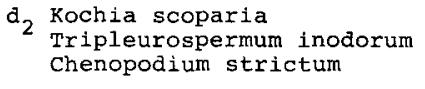 & • & $\begin{array}{l}\cdot \\
\cdot\end{array}$ & $\dot{\bullet}$ & $\begin{array}{l}\dot{\bullet} \\
\dot{\cdot}\end{array}$ & $\begin{array}{l}\dot{\bullet} \\
\dot{ }\end{array}$ & $\begin{array}{l}\cdot \\
\cdot\end{array}$ & $\begin{array}{l}\dot{\cdot} \\
\dot{ }\end{array}$ & {$\left[\begin{array}{l}3.2 \\
2.2 \\
.\end{array}\right.$} & $\begin{array}{l}1.2 \\
2.2 \\
2.2 \\
\end{array}$ & $\begin{array}{l}2.2 \\
2.2 \\
1.2 \\
\end{array}$ \\
\hline Sisymbrion-Arten & & & & & & & & & & \\
\hline $\begin{array}{l}\text { Conyza canadensis } \\
\text { Ambrosia artemisiifolia } \\
\text { Bromus sterilis } \\
\text { Malva neglecta } \\
\text { Descurainia sophia } \\
\text { Lactuca serriola }\end{array}$ & $\begin{array}{l}\dot{+} \\
\dot{\cdot} \\
\dot{\cdot}\end{array}$ & $\begin{array}{l}1.2 \\
. \\
:\end{array}$ & $\stackrel{\cdot}{.} \cdot 2$ & $\begin{array}{l}1.2 \\
. \\
. \\
. \\
.\end{array}$ & $\begin{array}{l}+ \\
2.2 \\
+. \\
. \\
.\end{array}$ & $\begin{array}{c}1.2 \\
. \\
. \\
. \\
.\end{array}$ & $\begin{array}{l}\cdot \\
\cdot \\
. \\
. \\
. \\
\cdot\end{array}$ & $\begin{array}{l}1.2 \\
. \\
. \\
. \\
.\end{array}$ & $\begin{array}{c}+ \\
. \\
1.2 \\
.\end{array}$ & $\begin{array}{c}: \\
: \\
\dot{1} \\
1.2\end{array}$ \\
\hline Chenopodietea-Arten & & & & & & & & & & \\
\hline $\begin{array}{l}\text { Atriplex patula } \\
\text { Setaria viridis } \\
\text { Amaranthus retroflexus } \\
\text { Sonchus oleraceus } \\
\text { Chenopodium album } \\
\text { Sonstige }\end{array}$ & $\begin{array}{l}\dot{.} \\
\dot{.} \\
\dot{.}\end{array}$ & $\begin{array}{l}\dot{.} \\
\dot{.} \\
\dot{.}\end{array}$ & $\begin{array}{c}. \\
+.2 \\
.\end{array}$ & $\begin{array}{l}+ \\
+ \\
\dot{.} \\
\dot{.}\end{array}$ & $\begin{array}{c}\dot{1} \\
\dot{.} \\
\dot{ }\end{array}$ & $\begin{array}{c}+ \\
+ \\
2.2 \\
.\end{array}$ & $\begin{array}{c}3.4 \\
. \\
. \\
.\end{array}$ & $\begin{array}{c}2.3 \\
: \\
1.1\end{array}$ & $\begin{array}{c}2.2 \\
\cdot \\
\cdot \\
\cdot\end{array}$ & $\begin{array}{l}+ \\
\dot{.} \\
\dot{\cdot}\end{array}$ \\
\hline $\begin{array}{l}\text { Polygonum aviculare agg. } \\
\text { Artemisia vulgaris juv. } \\
\text { Agropyron repens } \\
\text { Achillea millefolium agg. }\end{array}$ & $\begin{array}{l}2.2 \\
+ \\
1.2 \\
1.2\end{array}$ & $\begin{array}{l}2.1 \\
1.2\end{array}$ & $\begin{array}{c}1.2 \\
: \\
:\end{array}$ & $\dot{+}$ & $\begin{array}{c}2.3 \\
1.2 \\
:\end{array}$ & $\begin{array}{c}2.3 \\
: \\
\bullet\end{array}$ & $\begin{array}{c}+ \\
+ \\
1.2\end{array}$ & $\begin{array}{c}1.2 \\
1.1 \\
1.1 \\
.\end{array}$ & $\begin{array}{c}1.2 \\
1.1 \\
.\end{array}$ & $\begin{array}{c}1.1 \\
+ \\
1.2 \\
.\end{array}$ \\
\hline \multicolumn{11}{|c|}{$\begin{array}{l}\text { AuBerdem in Nr. 2: } 2.2 \text { Cichorium intybus, + Plantago major. Nr.5: +.2 Ballota } \\
\text { nigra ssp. nigra, + }{ }^{\circ} \text { Conium maculatum. Nr. 6: +.2 Urtica dioica, + Zea mays. } \\
\text { Nr. 8: + Daucus carota. Nr. 9: } 1.2 \text { Cardus acanthoides, } 1.2 \text { Cirsium arvense, + } \\
\text { Diplotaxis tenuifolia, + Convolvulus arvensis. Nr. 10: } 1.2 \text { Lepldium ruderale, } \\
+ \text { Consolida regalis, + }{ }^{\circ} \text { Rumex patientia. }\end{array}$} \\
\hline
\end{tabular}




\subsubsection{Malvetum pusillae Morariu 1943 (Tab. 6)}

Das $M$ a $1 \mathrm{v}$ e $\mathrm{t} \mathrm{u} \mathrm{m}$ pu s illa e ist eine artenarme und durch Dominanz von Malva pusilla charakterisierte osteuropäische Pflanzengesellschaft. Ihre Standortsbedingungen werden treffend von KRIPPELOVÁ (1981) charakterisiert: „Die Bestände mit $\mathrm{M}$ a l v e $\mathrm{t} \mathrm{u} \mathrm{m}$ p u sill a e kommen in Höfen mit Geflügelzucht und in der Nähe von Düngerhaufen vor. Häufig knüpfen sie an Trittgesellschaften der Klasse P l a n t a gi n e t e a". Auch bei den Aufnahmen von KRIPPELOVÁ (1981) erreichen die Trittpflanzen eine höhere Stetigkeit als die C h e n o p odi e t e a-Arten.

Tabelle 6. Malvetum pusillae Morariu 1943.

\begin{tabular}{|c|c|c|c|c|c|c|c|}
\hline Nummer & der Aufnahme & 1 & 2 & 3 & 4 & 5 & 6. \\
\hline Fläche & $\left(m^{2}\right)$ & 4 & 2 & 3 & 1 & 4 & 1,5 \\
\hline Veget: & =ionsbedeckung $(8)$ & 90 & 60 & 95 & 90 & 100 & 100 \\
\hline Artenz & hl & 16 & 12 & 10 & 9 & 16 & 11 \\
\hline $\mathrm{AC}$ & Malva pusilla & 5.5 & 4.5 & 5.5 & 5.5 & 5.5 & 5.5 \\
\hline $\mathrm{VC}-\mathrm{KC}$ & $\begin{array}{l}\text { Amaranthus retroflexus } \\
\text { Lepidium ruderale } \\
\text { Amaranthus chlorostachys } \\
\text { Stellaria media } \\
\text { Chenopodium album } \\
\text { Urtica urens } \\
\text { Chenopodium hybridum } \\
\text { Capsella bursa-pastoris } \\
\text { Mercurialis annua } \\
\text { Atriplex patula } \\
\text { Solanum nigrum } \\
\text { Conyza canadensis }\end{array}$ & $\begin{array}{l}+ \\
+ \\
r \\
+ \\
+ \\
+ \\
\dot{.} \\
\dot{.} \\
\dot{.} \\
\dot{.}\end{array}$ & $\begin{array}{l}r \\
+ \\
r \\
\dot{+} \\
\dot{r} \\
r \\
\dot{r} \\
\dot{.} \\
\dot{ }\end{array}$ & 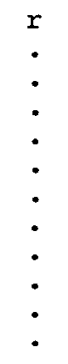 & $\begin{array}{l}+ \\
\dot{.} \\
\dot{.} \\
\dot{.} \\
\dot{ } \\
\dot{+} \\
+ \\
\mathrm{r} \\
\dot{ }\end{array}$ & $\begin{array}{l}\mathrm{r} \\
+ \\
\dot{.} \\
\dot{\mathrm{r}} \\
\dot{.} \\
\dot{.} \\
\dot{\mathrm{r}}\end{array}$ & $\begin{array}{l}\dot{+} \\
+ \\
\dot{.} \\
\dot{.} \\
\dot{r} \\
\dot{.} \\
\dot{.}\end{array}$ \\
\hline 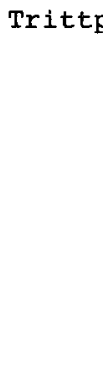 & $\begin{array}{l}\text { Elanzen und Grünlandarten: } \\
\text { Polygonum aviculare agg. } \\
\text { Plantago major } \\
\text { Taraxacum officinale agg. } \\
\text { Poa annua } \\
\text { Plantago lanceolata } \\
\text { Lolium perenne } \\
\text { Potentilla anserina } \\
\text { Trifolium repens } \\
\text { Achillea millefolium agg. } \\
\text { Pastinaca sativa } \\
\text { Dactylis glomerata }\end{array}$ & $\begin{array}{c}2.3 \\
1.2 \\
+ \\
+.2 \\
r \\
. \\
. \\
. \\
.\end{array}$ & $\begin{array}{c}2.3 \\
r \\
r \\
. \\
. \\
. \\
. \\
.\end{array}$ & $\begin{array}{c}2.3 \\
+ \\
+ \\
+.2 \\
+.2 \\
1.2 \\
+.2 \\
. \\
.\end{array}$ & 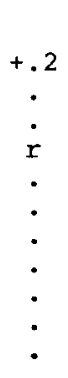 & $\begin{array}{c}+.2 \\
r \\
1.2 \\
\dot{1} .2 \\
2.2 \\
+.2 \\
+.2 \\
\dot{r} \\
.\end{array}$ & $\begin{array}{l}2.2 \\
\dot{r} \\
+.2 \\
\cdot \\
2.2 \\
\dot{.} \\
\dot{.}\end{array}$ \\
\hline Sonst & ge: & & & & & & \\
\hline & $\begin{array}{l}\text { Arctium minus } \\
\text { Chenopodium glaucum } \\
\text { Ballota nigra } \\
\text { Triticum aestivum Keiml. } \\
\text { Geranium spec. Keiml. } \\
\text { Artemisia vulgaris }\end{array}$ & $\begin{array}{l}r \\
+ \\
+.2 \\
\dot{r} \\
\dot{ } \\
\dot{ }\end{array}$ & $\begin{array}{l}\dot{+} \\
\dot{r} \\
\dot{.} \\
\dot{.}\end{array}$ & $\begin{array}{l}\dot{r} \\
\dot{.} \\
\dot{.} \\
\dot{.}\end{array}$ & $\begin{array}{l}1.1 \\
+.2 \\
+ \\
:\end{array}$ & $\begin{array}{l}\dot{r} \\
\dot{r} \\
\dot{r}\end{array}$ & $\begin{array}{l}\dot{ } \\
\dot{r} \\
\dot{r} \\
+.2\end{array}$ \\
\hline
\end{tabular}




\subsubsection{Weitere Chenopodietea-Gesellschaften}

An den Rändern der Scheunengassen und Durchgangsstraßen finden sich oft durch thermophile Arten gekennzeichnete Unkrautbestände, die teils zum $\mathrm{C}$ h e n op odie t u m ruderale (Tab. 7), teils zum Eragrostion gehören mögen (Tab. 8). Wichtige Arten sind Amarantbus retroflexus, Amarantbus albus agg., Chenopodium strictum, Digitaria sanguinalis, Eragrostis minor, Mercurialis annua, Portulaca oleracea und Solanum nigrum.

Tabelle 7. Chenopodietum ruderale Oberd. 1957.

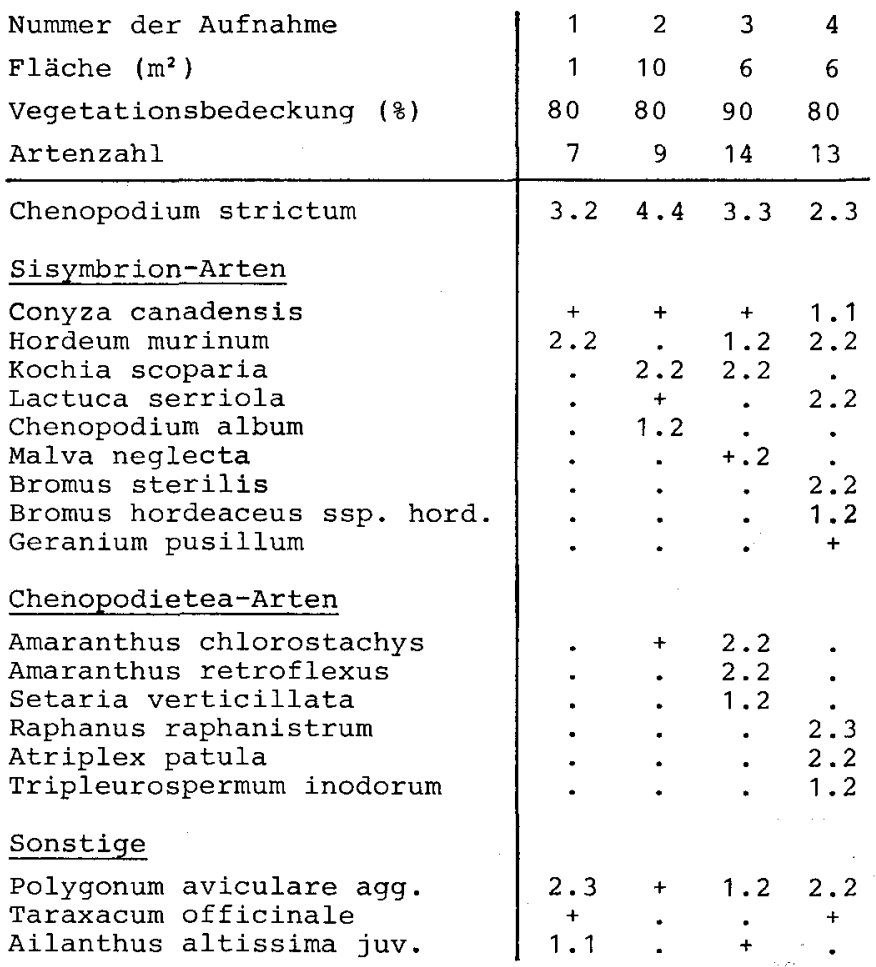

AuBerdem in Nr. 1: + Lepidium ruderale. Nr. 2: + Artemisia vulgaris juv., + Medicago lupulina. Nr. 3: 2.3 Convolvulus arvensis, 2.3 Urtica dioica, + Carduus acanthoides, + Sambucus nigra juv. Nr. 4: 1.2 Inula britannica.

Vergleichsweise selten scheint das A t $r$ i p 1 i c e $t$ u a $\mathrm{cuminat}$ a Knapp $1945 \mathrm{zu}$ sein; es wurde nur an wenigen ortsnahen Schuttplätzen angetroffen. 
Tabelle 8. Chenopodietea-Bestände.

\begin{tabular}{|c|c|c|c|c|c|c|}
\hline Nummer der Aufnahme & 1 & 2 & 3 & 4 & 5 & 6 \\
\hline Fläche $\left(m^{2}\right)$ & 20 & 30 & 15 & 11 & 16 & 4 \\
\hline Vegetationsbedeckung ( $(8)$ & 98 & 80 & 90 & 70 & 65 & 70 \\
\hline Artenzahl & 21 & 18 & 21 & 12 & 11 & 8 \\
\hline $\begin{array}{l}\text { Chenopodium album } \\
\text { Amaranthus retroflexus } \\
\text { Galinsoga parviflora }\end{array}$ & $\begin{array}{l}4.4 \\
2.2 \\
+\end{array}$ & $\begin{array}{l}3 \cdot 3 \\
2.3 \\
+\end{array}$ & $\begin{array}{l}3.3 \\
2.2 \\
1.2\end{array}$ & $\begin{array}{l}2.2 \\
3.3 \\
2.2\end{array}$ & $\begin{array}{l}2.1 \\
1.2 \\
.\end{array}$ & $\begin{array}{l}1.2 \\
1.2 \\
1.2\end{array}$ \\
\hline $\begin{array}{l}\text { Malva neglecta } \\
\text { Capsella bursa-pastoris } \\
\text { Stellaria media } \\
\text { Senecio vulgaris } \\
\text { Chenopodium hybridum }\end{array}$ & $\begin{array}{c}2.2 \\
+ \\
\dot{2} \\
\dot{2}\end{array}$ & $\begin{array}{l}\dot{\cdot} \\
\dot{+} \\
+ \\
1.1\end{array}$ & $\begin{array}{l}1.2 \\
+ \\
+.2 \\
+.2 \\
1.1\end{array}$ & $\begin{array}{l}\dot{\bullet} \\
\dot{.} \\
\dot{+}\end{array}$ & $\begin{array}{l}\dot{.} \\
\dot{.} \\
\dot{\cdot}\end{array}$ & $\begin{array}{l}\dot{.} \\
\dot{.} \\
\dot{ }\end{array}$ \\
\hline $\begin{array}{l}\text { Portulaca oleracea } \\
\text { Digitaria sanguinalis } \\
\text { Eragrostis minor } \\
\text { Conyza canadensis }\end{array}$ & $\begin{array}{l}+ \\
\dot{ } \\
\dot{ }\end{array}$ & $\begin{array}{l}\dot{ } \\
\dot{\cdot}\end{array}$ & $\dot{\bullet} \cdot \dot{ }$ & $\begin{array}{l}3.3 \\
1.2 \\
. \\
\text {. }\end{array}$ & $\begin{array}{c}3.2 \\
+ \\
.\end{array}$ & $\begin{array}{l}3.2 \\
2.1 \\
1.2 \\
r\end{array}$ \\
\hline $\begin{array}{l}\text { Solanum nigrum } \\
\text { Mercurialis annua } \\
\text { Amaranthus chlorostachys } \\
\text { Atriplex patula } \\
\text { Sonchus oleraceus } \\
\text { Diplotaxis muralis } \\
\text { Chenopodium murale } \\
\text { Hordeum murinum } \\
\text { Lactuca serriola } \\
\text { Artemisia annua } \\
\text { Setaria glauca } \\
\text { Ambrosia artemisifolia } \\
\text { Datura stramonium }\end{array}$ & $\begin{array}{l}1.2 \\
2.2 \\
: \\
\dot{+} \\
+ \\
+ \\
+ \\
\dot{ } \\
\dot{ } \\
.\end{array}$ & $\begin{array}{l}1.2 \\
1.2 \\
3.3 \\
. \\
. \\
. \\
. \\
+ \\
. \\
. \\
.\end{array}$ & $\begin{array}{l}1.2 \\
\dot{+} \\
3.3 \\
\dot{ } \\
. \\
. \\
. \\
1.2 \\
+.2 \\
. \\
\cdot\end{array}$ & $\begin{array}{l}: \\
1.1 \\
1.2 \\
\therefore \\
\therefore \\
\therefore \\
\therefore \\
1.2 \\
.\end{array}$ & 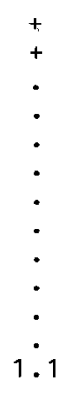 & 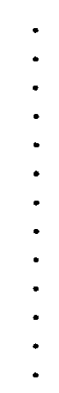 \\
\hline Begleiter & & & & & & \\
\hline $\begin{array}{l}\text { Agropyron repens } \\
\text { Echinochloa crus-galli } \\
\text { Taraxacum officinale } \\
\text { Chenopodium ficifolium }\end{array}$ & $\begin{array}{l}+ \\
+ \\
+ \\
+\end{array}$ & $\begin{array}{l}+ \\
1.2 \\
+\end{array}$ & $\begin{array}{l}1.2 \\
1.2 \\
1.1 \\
.\end{array}$ & $\begin{array}{c}1.2 \\
2.2 \\
. \\
.\end{array}$ & $\begin{array}{l}+ \\
\dot{+}\end{array}$ & $\dot{.}$ \\
\hline
\end{tabular}

AuBerdem in Nr. 1: + Poa annua, + Artemisia vulgaris juv., + ${ }^{\circ}$ Leonurus cardiaca, r Matricaria discoidea, r Plantago major. Nr. 2: + Galium aparine, + Hyoscyamus niger, + Onopordum acanthium, + Silene alba, $r$ Conium maculatum. Nr. 3: 2.2 Convolvulus arvensis, 1.2 Ballota nigra ssp. nigra, 1.2 Lolium perenne, + Rumex patientia, + Arctium spec. Nr. 4: + Erodium cicutarium. Nr. 5: + Polygonum aviculare agg., + Achillea millefolium agg.

Einzelaufnahme 6:

Schuttplatz bei Purbach. $40 \mathrm{~m}^{2}$, D $100 \% .14 .8 .1985$ :

AC: 5.5 Atriplex acuminata; VC-KC: 2.2 Tripleurospermum inodorum, 1.2 Lactuca serriola, + Descurainia sopbia + Lepidium campestre, 1.2 Chenopodium album, + Atriplex patula, + Amaranthus retroflexus; B: 1.2 Carduus acantboides, 1.2 Agropyron repens, +.2 Artemisia absintbium. 
4.3. Wärmebedürftige Distel-Gesellschaften (Onopordion acanthii Br.-Bl. 1926) 4.3.1. Onopordetum acanthii Br.-Bl. (1923 n.n.) 1926 (Tab. 9)

Das on opordet u a c a $\mathrm{m} \mathrm{hi}$ i ist eine charakteristische Pflanzengesellschaft der Dorfränder und Feldwege im nördlichen Burgenland. Die von uns aufgenommenen Bestände gehören sämtlich zur östlichen Rasse von Carduus acanthoides, möglicherweise zu einer eigenen (west)pannonischen Gebietsausbildung von Rumex patientia.

Je nach den standörtlichen Gegebenheiten lassen sich zwei Subassoziationen unterscheiden:

- Im Seewinkel gedeihen auf tiefgründigen, ausreichend mit Wasser und Nährstoffen versorgten „Böden" üppige, 1,50 bis $2 \mathrm{~m}$ hohe Eseslsdistel-Gestrüppe, an deren Aufbau auch Ballota nigra ssp. nigra, Artemisia vulgaris, Conium maculatum sowie Rumex patientia beteiligt sind. Diese Bestände vermitteln in ihrer Artenzusammensetzung und Wüchsigkeit zum A r c t i o $\mathrm{n}$, sie werden als O n o p ordetum a c a thii arctiet os um Brandes 1980 angesprochen (Tab. 9).

- Vorwiegend zwischen Neusiedler See und Leithagebirge finden sich auf skelettreichen und durchlässigen Böden nur ca. 1 bis $1,2 \mathrm{~m}$ hohe, jedoch auffällig bunt blühende Eselsdistel-Gestrüppe. Diese werden zur Subassoziation von Echium vulgare und Berteroa incana gestellt. Nomenklatorischer Typus der $\mathrm{Sub}$ a s oziation von Echium vulgare ist die folgende Aufnahme:

Einzelaufnahme 7:

Steinbruch St. Margarethen. $100 \mathrm{~m}^{2}$, D $100 \%$. 16.7.1975:

AC: 3.3 Onopordum acanthium; D: 2.2 Echium vulgare, 2.2 Berteroa incana; VC: 2.3 Carduus acantboides, 1.2 Daucus carota, +.2 Pastinaca sativa, +.2 Verbascum pblomoides, +.2 Melilotus officinalis, +.2 Reseda lutea, + Anchusa officinalis; OC/KC: 2.2 Silene alba, 1.2 Artemisia vulgaris; B: 2.2 Arrbenatberum elatius, 2.2 Dactylis glomerata, 2.2 Agropyron repens, 1.2 Tripleurospermum inodorum, 1.2 Convolvulus arvensis, 1.2 Centaurea stoebe, 1.2 Diplotaxis tenuifolia, +.2 Achillea millefolium agg., + +.2 Sisymbrium loeselii, +.2 Medicago falcata, + Papaver rboeas.

Dem O nopordet $\mathrm{u}$ m gehen meistens $\mathrm{S}$ is y mbrion-Ge se11$\mathrm{s} c \mathrm{~h}$ a $\mathrm{f} \mathrm{t} \mathrm{e} \mathrm{n}$ voraus, weswegen junge und/oder kleinflächige Bestände des O n o p o r d e t u m eine abweichende Artenzusammensetzung haben können.

Einzelaufnahme 8:

Frauenkirchen, südexponierter Mauerfuß. $8 \mathrm{~m}^{2}$, D $90 \%$. 13.8.1985:

AC: 3.2 Onopordum acantbium; KC: 2.3 Artemisia vulgaris; B: 3.2 Descurainia sophia, 2.2 Tripleurospermum inodorum, 1.2 Hordeum murinum, 1.2 Lolium perenne, 1.2 Agropyron repens, + Conium maculatum, + Lactuca serriola.

Wichtige Kontakt- bzw. Folgegesellschaften des $\mathrm{O} n$ op orde $\mathrm{t} u \mathrm{~m}$ a $\mathrm{c}$ a $\mathbf{n}$ thi arctietosum sind Arctio-Artemisietum, Conietu m $\mathrm{m}$ a $\mathrm{c}$ u $\mathrm{l}$ a $\mathrm{t} \mathrm{i}$ und Robinia pseudacacia-Ailantbus altissima-Gebüsche. Die Kontaktgesellschaften des on opordetum a canthi echietosum ge- 
Tabelle 9. Onopordetum acanthii Br.-Bl. (1923 n.n.) 1926 arctietosum Brandes 1980.

Nummer der Aufnahme
Fläche $\left(\mathrm{m}^{2}\right)$
Vegetationsbedeckung ( 8$)$
Exposition
Neigung $\left({ }^{\circ}\right)$
Artenzahl
AC Onopordetum acanthii
Onopordum acanthium
$\Delta$ Rasse von Carduus acanthoides

Carduus acanthoides

D Subassoziation bzw. Gebietsausb.

\section{Ballota nigra ssp. nigra \\ Conium maculatum \\ Rumex patientia \\ Galium aparine \\ Chelidonium majus \\ Arctium tomentosum \\ Arctium lappa \\ Leonurus cardiaca}

\section{VC Onopordion u. Dauco-Melilotion}

\section{Artemisia absinthium}

Verbascum phlomoides

Picris hieracioides

Melilotus officinalis

Echinops sphaerocephalus

\section{KC Artemisietea}

Artemisia vulgaris

Urtica dioica

Reynoutria japonica

Cirsium arvense

Silene alba

\section{Agropyretea-Arten}

\section{Agropyron repens}

Convolvulus arvensis

Bromus inermis

Cardaria draba

\section{Chenopodietea-Arten}

\section{Bromus sterilis}

Atriplex acuminata

Tripleurospermum inodorum

Chenopodium album

Sisymbrium altissimum

Descurainia sophia

Atriplex patula

Lactuca serriola

Bromus tectorum

\section{Sonstige}

Poa pratensis

Sambucus nigra

Dactylis glomerata

\section{$\begin{array}{llllllll}2.3 & 2.3 & 3.2 & 3.2 & 4.3 & 2.2 & 3.2\end{array}$}

$4.3 .3 .3+.2 .22 .3$

$\begin{array}{ccccccc}2.2 & 2.3 & 3.2 & 3.3 & 2.3 & 2.3 & 2.3 \\ 1.1 & 2.3 & 2.2 & : & 1.2 & 3.3 & : \\ 1.2 & : & 1.2 & : & 1.2 & 1.2 & 3.3 \\ 1.2 & : & \cdot & 2.2 & 1.2 & 3.3 & : \\ : & + & : & : & : & : & : \\ : & : & 1.1 & : & 1.1 & : & : \\ : & : & : & : & . & + & 1.2\end{array}$

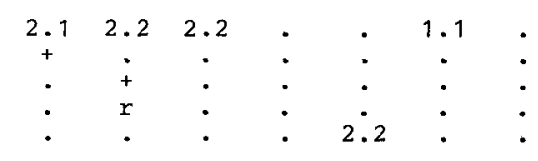

$\begin{array}{llllll}2.2 & 2.2 & 2.3 & 2.2 & 1.1 & 2.1\end{array}$.

$\begin{array}{ccccccc}2.2 & 2.2 & 2.3 & 2.2 & 1.1 & 2.1 \\ 2.2 & 1.2 & 1.2 & 2.2 & . & 3.3 & : \\ . & 2.2 & . & . & . & . & : \\ . & . & . & . & 1.1 & . & \text {. } \\ . & . & . & . & . & 1.2 & +\end{array}$

$\begin{array}{ccccccc}2.2 & 2.2 & 2.3 & 2.2 & 3.4 & 2.2 & 2.2 \\ 1.2 & + & : & : & 1.2 & 1.2 & 2.2 \\ 1.2 & +.2 & \cdot & : & . & . & : \\ . & 3.3 & \cdot & . & 1.2 & . & :\end{array}$

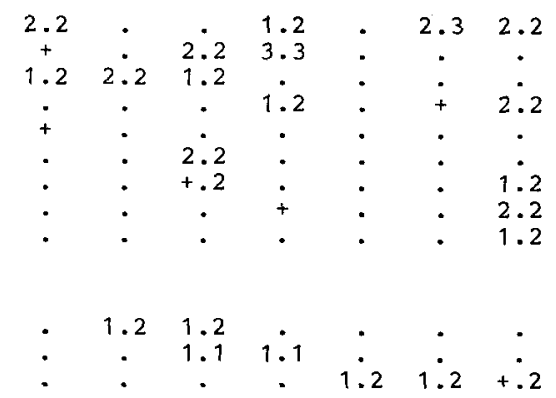

AuBerdem in Nr. 1: + Papaver rhoeas, 1.1 Seseli libanotis, 1.2 Keimlinge indet. $\mathrm{Nr} .2 \mathrm{2} 1.2$ Keimlinge indet. Nr. 4: 1.2 Bryonia dioica. $\mathrm{Nr} .5:+$ Verbena officinalis. Nr. 6: 1.1 Robinia pseudacacia juv., 2.2 Lycium barbarum, 1.2 Fallopia convolvulus, + Polygonum aviculare agg. Nr. 7 : + Arrhenatherum elatius. 
hören dagegen zu den ruderalen Trockenrasen. Der Grundstock an C o n v o l$\mathrm{v} u 1$ o- A g r o p y r i o $\mathrm{n}$-Arten ist bereits in der Gesellschaft vorhanden, bei mechanischen Störungen können diese Arten leicht zur Dominanz kommen.

\subsubsection{Marrubium vulgare-Gesellschaft (Tab. 10)}

Marrubium vulgare ist eine alte Heilpflanze, die in Wärme- und Trockengebieten Mitteleuropas eingebürgert, jedoch nur in Südeuropa einheimisch ist (HEGI 1975). Heute ist diese zoochore Art stark im Rückgang begriffen, so daß an dieser Stelle auf ihre Vorkommen in Dörfern des Seewinkels hingewiesen werden soll.

Auf Hühnerhöfen finden sich im Übergangsbereich von Trittrasen (incl. $M$ a 1 $v$ e $\mathrm{t} \mathrm{u} \mathrm{m} \mathrm{n} \mathrm{e} \mathrm{g} \mathrm{le} \mathrm{c} \mathrm{t} \mathrm{a} \mathrm{e} \mathrm{/} \mathrm{p} \mathrm{u} \mathrm{s} \mathrm{i} \mathrm{lla} \mathrm{e)} \mathrm{zu} \mathrm{ruderalen} \mathrm{Staudengesellschaften}$ charakteristische Marrubium vulgare-Bestände. Den stark gestörten Wuchsorten entsprechend lassen sich diese Bestände keiner Assoziation zuordnen. Die artenreicheren Ausbildungen enthalten deutlich mehr Tritt- und Flutrasenarten, während die $\mathrm{S}$ i s y $\mathrm{m} \mathrm{b}$ r i o $\mathrm{n}$-Arten sich vor allem in den artenärmeren Beständen finden.

\subsubsection{Basalgesellschaft Carduus acanthoides-[Onopordetalia] (Tab, 11)}

An den Dorfrändern haben sich großflächige Carduus acantboides-Gestrüppe entwickelt, denen sowohl Kennarten des $\mathrm{O}$ n o p o rde $\mathrm{t} \mathrm{u} \mathrm{m}$ als auch anderer $\mathrm{O} \mathrm{n}$ o $\mathrm{p}$ o $\mathrm{rd}$ i o $\mathrm{n}$-Assoziation fehlen. Da die Ordnungskennart Carduus acanthoides dominiert, ferner sowohl $\mathrm{D}$ a u co-M eli lot $\mathrm{i}$ on-als auch $\mathrm{O}$ n o$\mathrm{p}$ o $\mathrm{r} \mathrm{d}$ i o $\mathrm{n}$-Verbandskennarten vorhanden sind, können diese Bestände nur als Basalgesellschaft klassifiziert werden.

\subsubsection{Salvio-Marrubietum peregrini Mucina 1981 (Tab. 12)}

Das $\mathrm{S}$ a $\mathrm{l}$ vi o- $\mathrm{M}$ a r r u b i e $\mathrm{t} \mathrm{m}$ peregrin i ist eine seltene Ruderalgesellschaft des pannonischen Raumes, die sich an Ortsrändern, mitunter auch auf großen Freiflächen innerhalb der Dörfer findet. Das ostsubmediterran-mediterrane Marrubium peregrinum ist im Untersuchungsgebiet streng an diese Assoziation gebunden. Es lassen sich zwei Ausbildungen unterscheiden: Die $\mathrm{V}$ a $\mathrm{r}$ i a $\mathrm{n}$ t e von Ery $\mathrm{ng}$ i u $\mathrm{m}$ c a m pest re erinnert mit Marrubium peregrinum, Falcaria vulgaris und Eryngium campestre beim flüchtigen Hinschauen an eine Dornsteppe, wobei allerdings die Vegetationsdecke fast geschlossen ist. Die $\mathrm{Variante}$ von $\mathrm{H}$ ord e u murinum istärmeran Onopordionbzw. D a u c o-M e 1 il o t i o n-Kennarten. Je lückiger der Bestand ist, desto höher ist seine Artenzahl n. Zwischen der Vegetationsbedeckung und der Artenzahl besteht eine annähernd lineare Beziehung.

Das $\mathrm{S}$ a $1 \mathrm{v}$ i o- $\mathrm{M}$ a $\mathrm{r} \mathbf{r} \mathbf{u} \mathbf{b}$ i e $\mathrm{t} \mathbf{u} \mathrm{m}$ ist unseres Wissens bislang nur aus der angrenzenden Südslowakei bekannt (MUCINA 1981, ELIÁŠ 1981). 
Tabelle 10. Marrubium vulgare-Bestände.

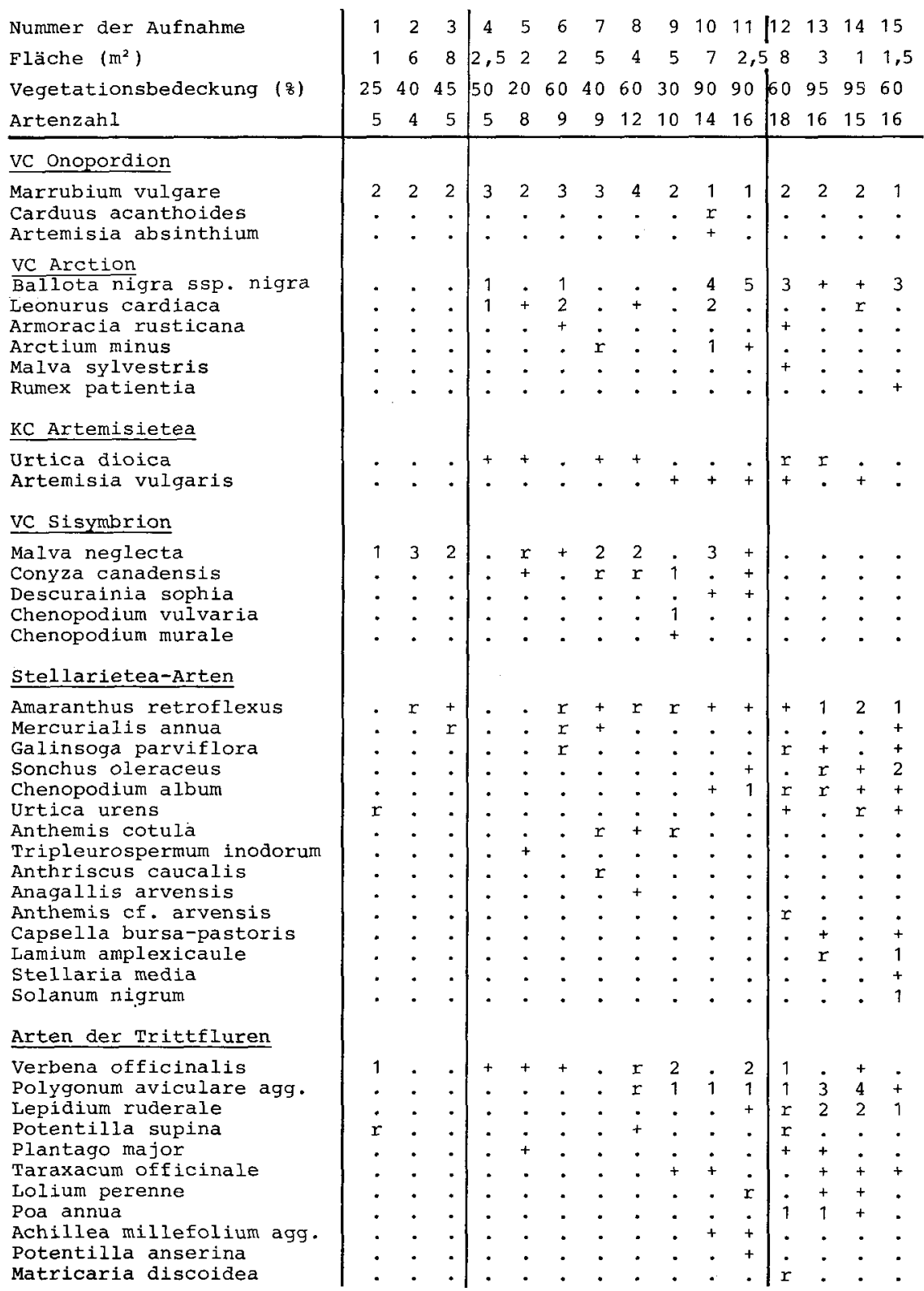

AuBerdem in Nr. 2: 1 Hedera helix (gepflanzt). Nr. 3: r Chenopodium glaucum. Nr. 8: r Chenopodium glaucum. Nr. 13: + Veronica spec. Nr. 14: + Convolvulus arvensis. 
Tabelle 11. Basalgesellschaft Carduus acanthoides-[Onopordetalia].

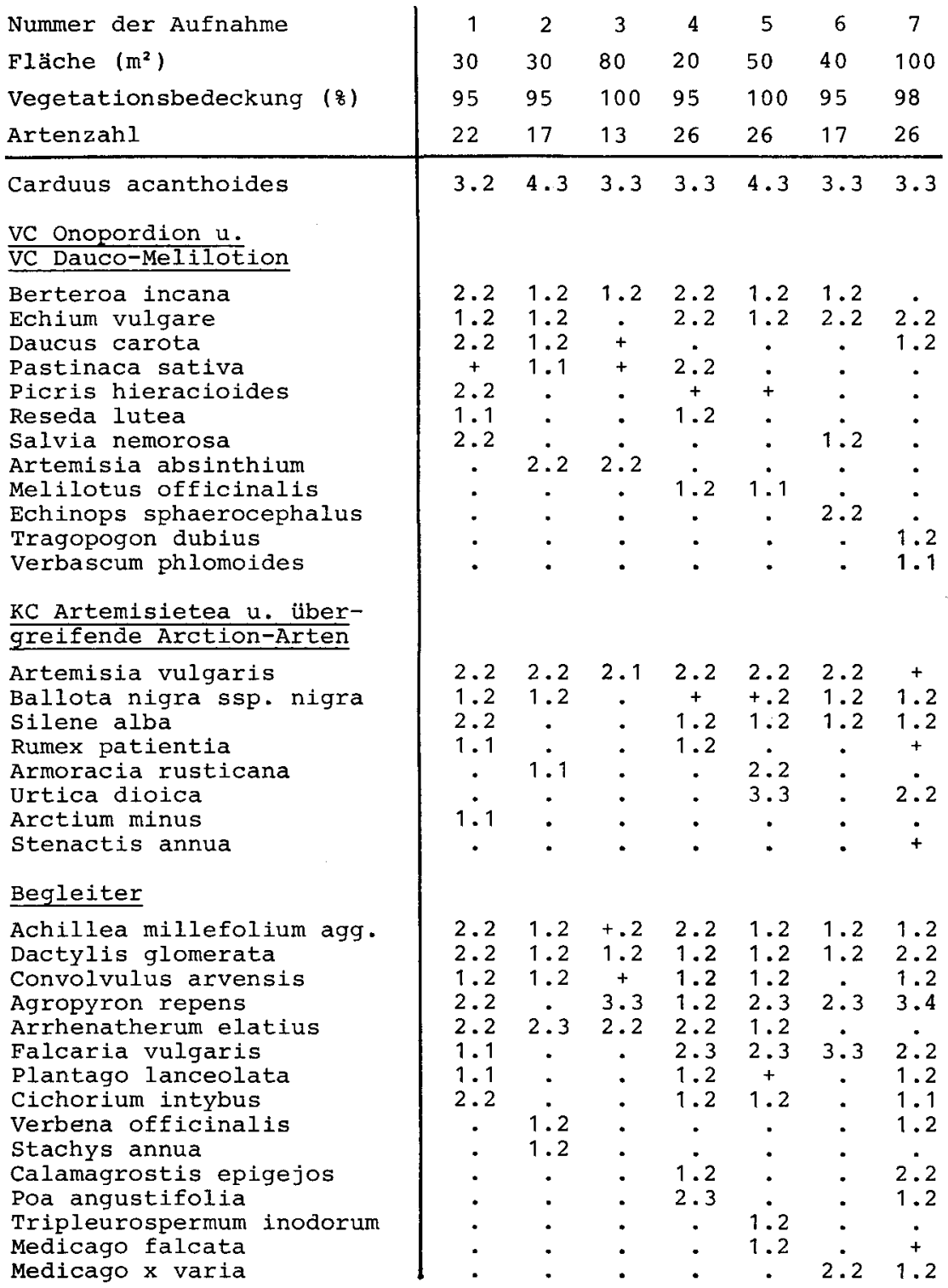

AuBerdem in Nr. 1: 1.2 Phleum pratense. Nr. 2: 2.3 Bromus sterilis, 1.1 Robinia pseudacacia juv. Nr. 3: 1.2 Galium verum, + Scabiosa ochroleuca. Nr. 4: 1.2 Lolium perenne, 1.2 Galium mollugo agg., 1.2 Coronilla varia, 1.1 Knautia arvensis, + Vicia cf. hirsuta. Nr. 5: 2.2 Centaurea scabiosa, 1.2 Alliaria petiolata, + Centaurea stoebe, + Lathyrus pratensis, + Fallopia convolvulus, r sisymbrium orientale. Nr. 6: 1.2 Poa pratensis, 1.2 Lycium barbarum, + Amaranthus hybridus, + Amaranthus retroflexus. Nr. 7: 1.2 Galium mollugo agg., + Lactuca serriola, $r$ Conyza canadensis. 
Tabelle 12. Salvio-Marrubietum peregrini Mucina 1981.

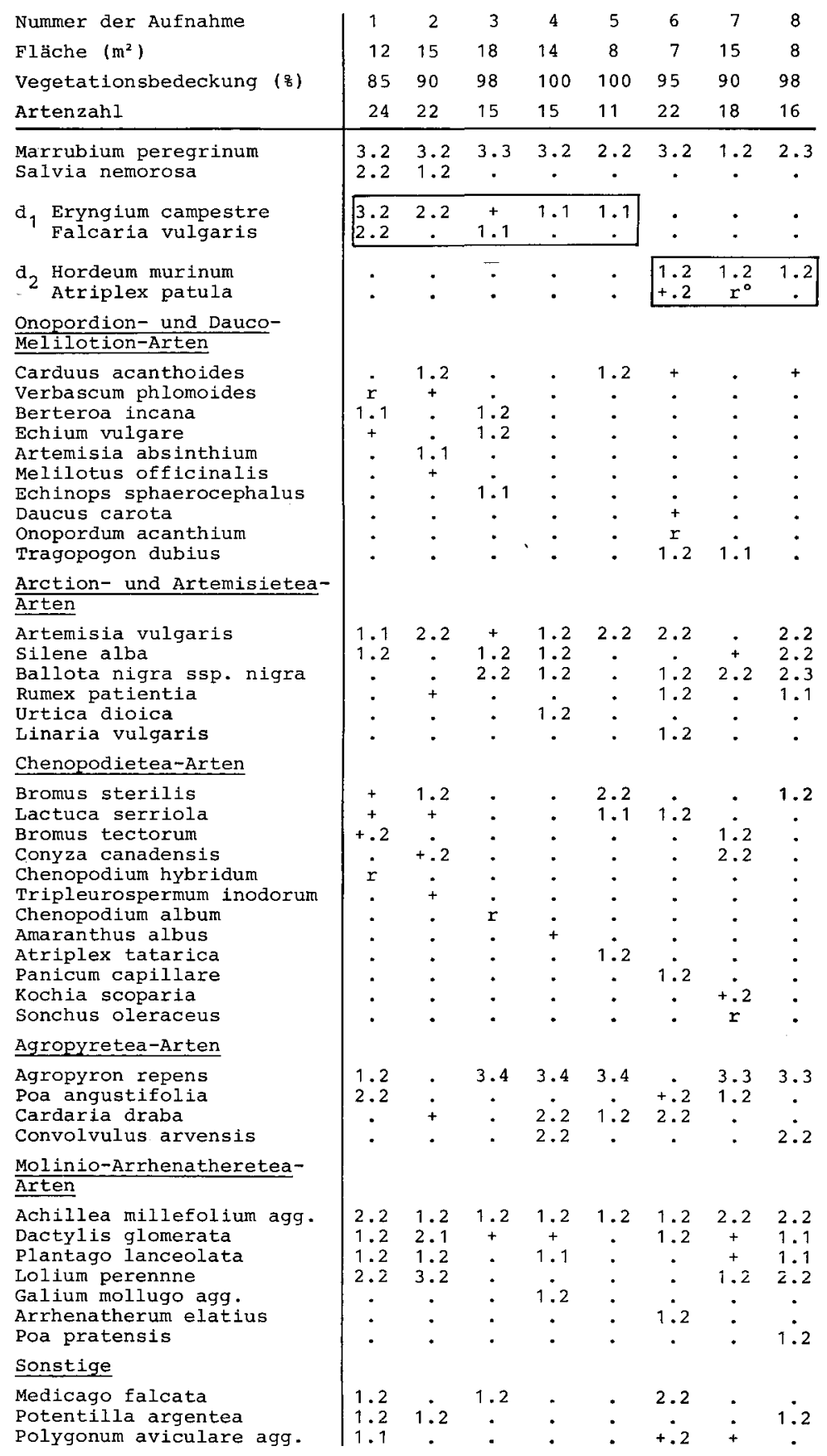

AuBerdem in Nr. 1: + Erodium cicutarium, + Centaurea stoebe. Nr. 2: 1.1 Medicago lupulina, +.2 Potentilla reptans, + Taraxacum officinale. Nr. 3: 1.1 Podospermum canum. Nr. 4: + Verbena officinalis. Nr. 5: 1.2 Cynodon dactylon. Nr. 6: 1.2 Diplotaxis tenuifolia, + Taraxacum officinale. Nr. 7: 1.1 Robinia pseudacacia juv. 


\subsubsection{Salvia nemorosa-Straßenrandgesellschaft (Tab. 13)}

Im nordöstlichen Burgenland gibt es besonders farbenprächtige Straßenränder. Gleichsam in die Rasenmatrix eingestreut finden sich Salvia nemorosa, Echium vulgare, Carduus acantboides, Pastinaca sativa, Berteroa incana und Silene alba. Die pflanzensoziologische Zuordnung ist problematisch, man muß diese Bestände als Mischung aus On o pordetalia-, Molin io-Arrhenathe$r$ e $t$ e a - und A g r o p y ret e a-Arten ansehen, die sich mit Hilfe eines Mischungsdreiecks gut darstellen läßt.

Ahnliche Straßenrandgesellschaften sind auch aus dem niederösterreichischen Weinviertel und aus der Wachau bekannt (BRANDES 1985).

\subsubsection{Potentillo argenteae-Artemisietum absinthii Faliński 1965 (Tab. 14)}

Wermut-Fluren gehören zur charakteristischen Vegetation der Dörfer des Seewinkels. An den Ortsrändern baut Artemisia absinthium dichte, silbrig-graue Bestände auf, in denen Centaurea stoebe ihren Verbreitungsschwerpunkt hat. Die Wermut-Fluren stehen häufig in Kontakt zu ruderalen Trockenrasen, an steilen, südexponierten Böschungen treten als seltene Arten Linaria genistifolia und Artemisia austriaca hinzu.

Artemisia absintbium, ,eine alte Heil- und Gewürzpflanze eurasiatisch-kontinental-submediterraner Verbreitung, häuft sich in Mitteleuropa in Trockengebieten. Die Syntaxonomie von Artemisia absintbium-Beständen erscheint diffizil, da Artemisia absintbium (a) faziesbildend auftritt, (b) ihre gehäuften Vorkommen in den Trockengebieten weit voneinander entfernt sind, und der Gesellschaftsanschluß deshalb unterschiedlich ist. Aus Polen und der Tschechoslowakei ist das Potentillo argenteae-Artemisiet um absinthii beschrieben, das in verarmter Form auch im nördlichen Deutschland auftritt (BRANDES 1985 a). Aus dem Unterengadin wurde das A r te mis i o-Agropyret u m Br.-Bl. 1949, aus dem Aostatal das E c h i n op s e t o-A r te m is ie t u m $\mathrm{Br}$-Bl. 1961 beschrieben. Wenn auch eine Zusammenfassung aller Wermutfluren naheliegend ist, so ist die Aufstellung der Klasse A r t e m is i e te a a b s i nt h i i Eliás 1981 noch verfrüht und wohl auch nicht haltbar. Auf jeden Fall ist eine Übersicht über die Artemisia absintbium-Gesellschaften Mitteleuropas dringend erforderlich.

Unsere Übersichtstabelle der o n o p or d i o n -Gesellschaften des Seewinkels (Tab. 15) läßt deutlich die Probleme der Abgrenzung der Verbände O n o p or d i o n und D a c o-M e li 1 ot i o n erkennen: Im nördlichen Burgenland greifen $\mathrm{D}$ a $\mathrm{u}$ c o- $\mathrm{M}$ e 1 i 1 o $\mathrm{t}$ i o $\mathrm{n}$-Arten auf durchlässigen Substraten regelmäßig in das $\mathrm{O} n$ o p ordion über; lediglich auf frischen und sehr nährstoffreichen Standorten fehlen die $D$ a u c o-M e 1 i 1 o t i o n-Arten, so im O n o p orde t u m a $\mathrm{cthi}$ a r ctiet os um und in der Marrubium vulgare-Gesellschaft. Umgekehrt sind die z.T. zoochoren $\mathrm{O}$ n o p o r d i o n Arten jedoch kaum in $\mathrm{D}$ a u c o-M elil o t i o $\mathrm{n}$-Gesellschaften anzutreffen. Ähnliche Beobachtungen gelten auch für das östliche Ungarn oder für das östliche Niedersachsen, wo eine floristisch saubere Trennung zwischen D a u coMelilotion und on o p ordion ebenfalls kaum möglich ist. 
Tabelle 13. Salvia nemorosa-Straßenrandgesellschaft.

\begin{tabular}{|c|c|c|c|c|c|c|}
\hline Nummer der Aufnahme & 1 & 2 & 3 & 4 & 5 & 6 \\
\hline Fläche $\left(\mathrm{m}^{2}\right)$ & 20 & 40 & 40 & 30 & 20 & 40 \\
\hline Vegetationsbedeckung (z) & 95 & 98 & 90 & 98 & 90 & 95 \\
\hline Artenzahl & 21 & 22 & 21 & 24 & 22 & 22 \\
\hline $\begin{array}{l}\text { Salvia nemorosa } \\
\text { Onopordion- bzw. Dauco- } \\
\text { Melilotion-Arten }\end{array}$ & 3.3 & 3.3 & 2.2 & 2.2 & 2.2 & 3.3 \\
\hline $\begin{array}{l}\text { Echium vulgare } \\
\text { Carduus acanthoides } \\
\text { Pastinaca sativa } \\
\text { Berteroa incana } \\
\text { Daucus carota } \\
\text { Tanacetum vulgare } \\
\text { Reseda lutea } \\
\text { Picris hieracioides } \\
\text { Artemisietalia- bzw. } \\
\text { Artemisietea-Arten }\end{array}$ & $\begin{array}{l}+ \\
1.1 \\
1.1 \\
\dot{+} \\
\dot{.} \\
.\end{array}$ & $\begin{array}{l}+ \\
1.1 \\
2.2 \\
. \\
\dot{.} \\
\dot{.}\end{array}$ & $\begin{array}{l}1.2 \\
: \\
2.3 \\
\dot{+} \\
\dot{-}\end{array}$ & $\begin{array}{l}1.2 \\
2.1 \\
1.2 \\
: \\
2.2 \\
.\end{array}$ & $\begin{array}{l}2.2 \\
+ \\
\cdot \\
\dot{ } \\
\dot{1} \\
1.2 \\
1.2\end{array}$ & $\begin{array}{l}+ \\
1.2 \\
2.2 \\
1.2 \\
1.1 \\
. \\
.\end{array}$ \\
\hline $\begin{array}{l}\text { Silene alba } \\
\text { Artemisia vulgaris } \\
\text { Ballota nigra ssp. nigra } \\
\text { Molinio-Arrhenatheretea- } \\
\text { Arten }\end{array}$ & $\begin{array}{l}+ \\
\dot{*}\end{array}$ & +.2 & $\begin{array}{l}1.2 \\
1.1 \\
.\end{array}$ & $\begin{array}{l}1.2 \\
1.1 \\
.\end{array}$ & $\begin{array}{l}1.2 \\
+\circ\end{array}$ & $\begin{array}{l}1.2 \\
1.2 \\
.\end{array}$ \\
\hline $\begin{array}{l}\text { Arrhenatherum elatius } \\
\text { Achillea millefolium agg. } \\
\text { Cichorium intybus } \\
\text { Plantago lanceolata } \\
\text { Lolium perenne } \\
\text { Taraxacum officinale } \\
\text { Galium mollugo agg. } \\
\text { Trifolium repens } \\
\text { Centaurea jacea agg. } \\
\text { Trifolium pratense } \\
\text { Knautia arvensis } \\
\text { Crepis biennis } \\
\text { Festuca rubra agg. } \\
\text { Dactylis glomerata } \\
\text { Agropyretea-Arten }\end{array}$ & $\begin{array}{c}2.2 \\
2.2 \\
2.1 \\
+ \\
2.2 \\
1.1 \\
1.2 \\
1.2 \\
. \\
. \\
. \\
. \\
.\end{array}$ & $\begin{array}{c}1.2 \\
2.2 \\
1.2 \\
+ \\
2.3 \\
+ \\
2.2 \\
1.2 \\
1.2 \\
. \\
. \\
. \\
.\end{array}$ & $\begin{array}{c}2.2 \\
2.2 \\
2.2 \\
2.2 \\
1.2 \\
1.1 \\
. \\
. \\
. \\
. \\
. \\
. \\
.\end{array}$ & $\begin{array}{c}3.3 \\
1.2 \\
1.1 \\
2.2 \\
1.2 \\
. \\
. \\
\dot{1} \\
+2 \\
+ \\
. \\
.\end{array}$ & 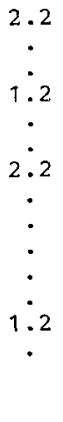 & $\begin{array}{c}3.2 \\
1.2 \\
\cdot \\
. \\
. \\
. \\
. \\
. \\
. \\
. \\
. \\
+\end{array}$ \\
\hline $\begin{array}{l}\text { Falcaria vulgaris } \\
\text { Convolvulus arvensis } \\
\text { Agropyron repens } \\
\text { Poa angustifolia } \\
\text { Sonstige }\end{array}$ & $\begin{array}{r}1.2 \\
+.2 \\
. \\
.\end{array}$ & $\begin{array}{c}1.1 \\
. \\
.\end{array}$ & $\begin{array}{c}1.2 \\
1.2 \\
. \\
.\end{array}$ & $\stackrel{+}{1.2}$ & $\begin{array}{c}2.2 \\
1.2\end{array}$ & $\begin{array}{c}1.2 \\
2.3 \\
1.2\end{array}$ \\
\hline $\begin{array}{l}\text { Conyza canadensis } \\
\text { Podospermum canum } \\
\text { Cynodon dactylon } \\
\text { Medicago lupulina } \\
\text { Eryngium campestre } \\
\text { Medicago falcata } \\
\text { Sisymbrium orientale } \\
\text { Silene vulgaris }\end{array}$ & $\begin{array}{c}\mathbf{r} \\
+ \\
2 \cdot 3 \\
. \\
. \\
.\end{array}$ & $\begin{array}{l}\dot{.} \\
2.3 \\
+ \\
1.2 \\
. \\
.\end{array}$ & $\begin{array}{c}+.2 \\
. \\
+.2 \\
+.2 \\
+ \\
+\end{array}$ & $\begin{array}{c}. \\
+.2 \\
\dot{.} \\
\dot{.} \\
+.2\end{array}$ & $\begin{array}{c}+ \\
+ \\
\dot{1} \\
\dot{1} \\
\dot{1} \\
1.2\end{array}$ & $\begin{array}{c}r \\
+ \\
\dot{ } \\
\dot{+} \\
2.2 \\
1.2 \\
.\end{array}$ \\
\hline
\end{tabular}

AuBerdem in $\mathrm{Nr} .1: 1.2$ Scabiosa ochroleuca, 1.2 Coronilla varia. $\mathrm{Nr}$. 2: 1.2 Pimpinella saxifraga, 1.2 Thymus pulegioides agg., + Potentilla argentea, + Lathyrus tuberosus. Nr. 3: 1.2 Plantago major, 1,2 Verbena officinalis, + Tripleurospermum inodorum. Nr. 4: 2.3 Bromus erectus, 2.2 Poa annua, 1.2 Stachys annua, 1.1 Centaurea scabiosa, +.2 Anagallis arvensis. Nr. 5: 2.2 Astragalus onobrychis, 2.2 Botriochloa ischaemum, 2.2 Medicago $x$ varia, 1.2 Thymus pulegioides agg., + Erodium cicutarium. Nr. 5: 1.2 Galium verum, +.2 Atriplex patula, I Lactuca serriola. 
Tabelle 14. Potentillo-Artemisietum absinthii Faliński 1965.

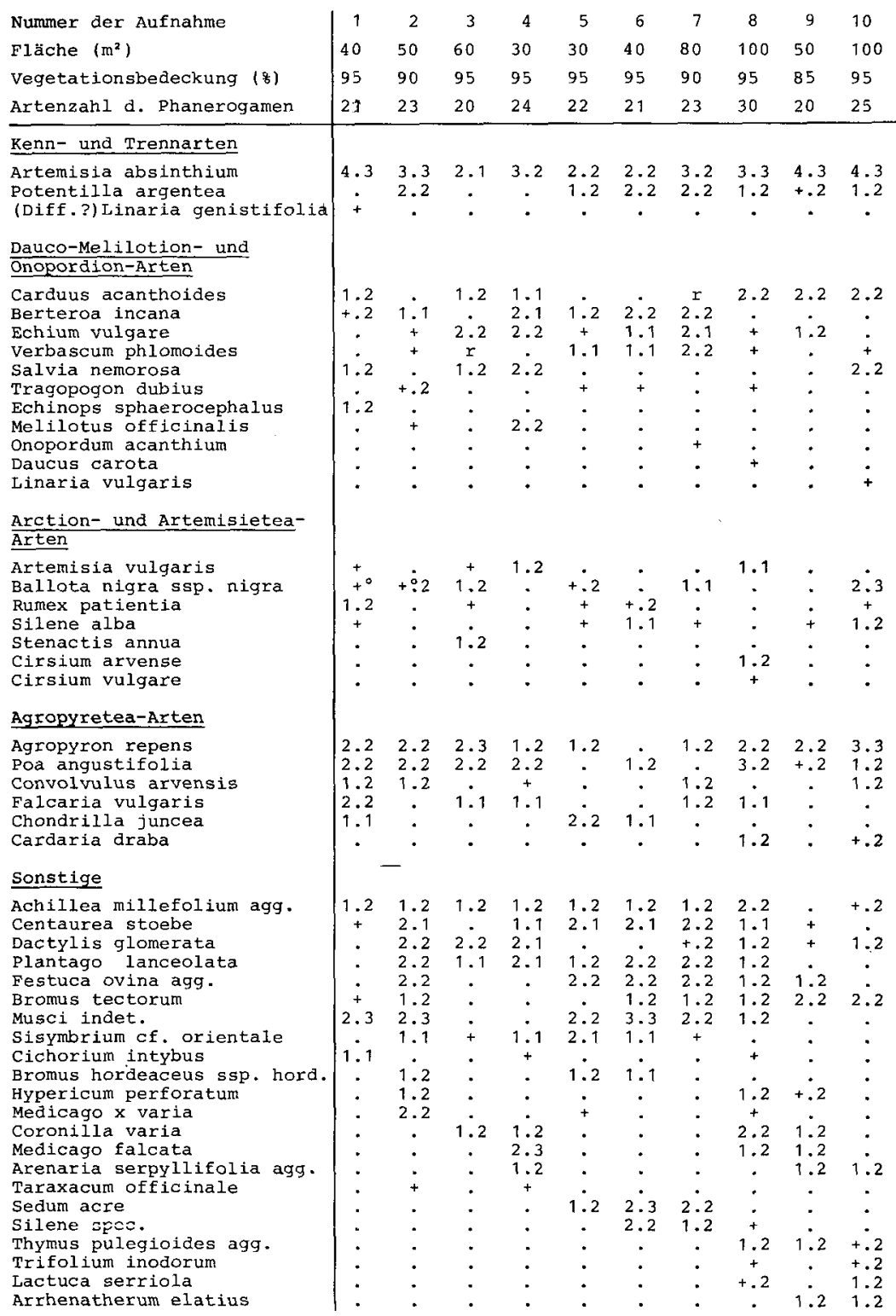

AuBerdem in Nr. 1: 1.1 Artemisia austriaca, 1.1 Artemisia campestris. Nr.2: +.2 Petrorhagia saxifraga. Nr. 3: 1.1 Eryngium campestre, 1,1 Lycium barbarum, + Calamintha clinopodium. $\mathrm{Nr}$. 4: 1.2 Eryngium campestre, 1.2 Medicago lupulina, + Conyza canadensis. Nr. 5: 2.2 Petrorhagia saxifraga, 1.1 Eryngium campestre. $\mathrm{Nr}, 6: 2.1$ Petrorhagia saxifraga, + Eryngium campestre. Nr. 7: 1.2 Petrorhagia saxifraga, 2.2 Astragalus onobrychis. Nr. 8: + Trifolium spec. Nr. 9: 1.2 GaIlum verum, + Chenopodium album, r Stenactis annua. Nr. 10: 2.3 Anthriscus cerefolium, + Eryngium campestre, + Sisymbrium altissimum, 1.2 Trifolium spec. 
Tabelle 15. Ubersichtstabelle der Onopordion-Gesellschaften des nordöstlichen Burgenlandes.

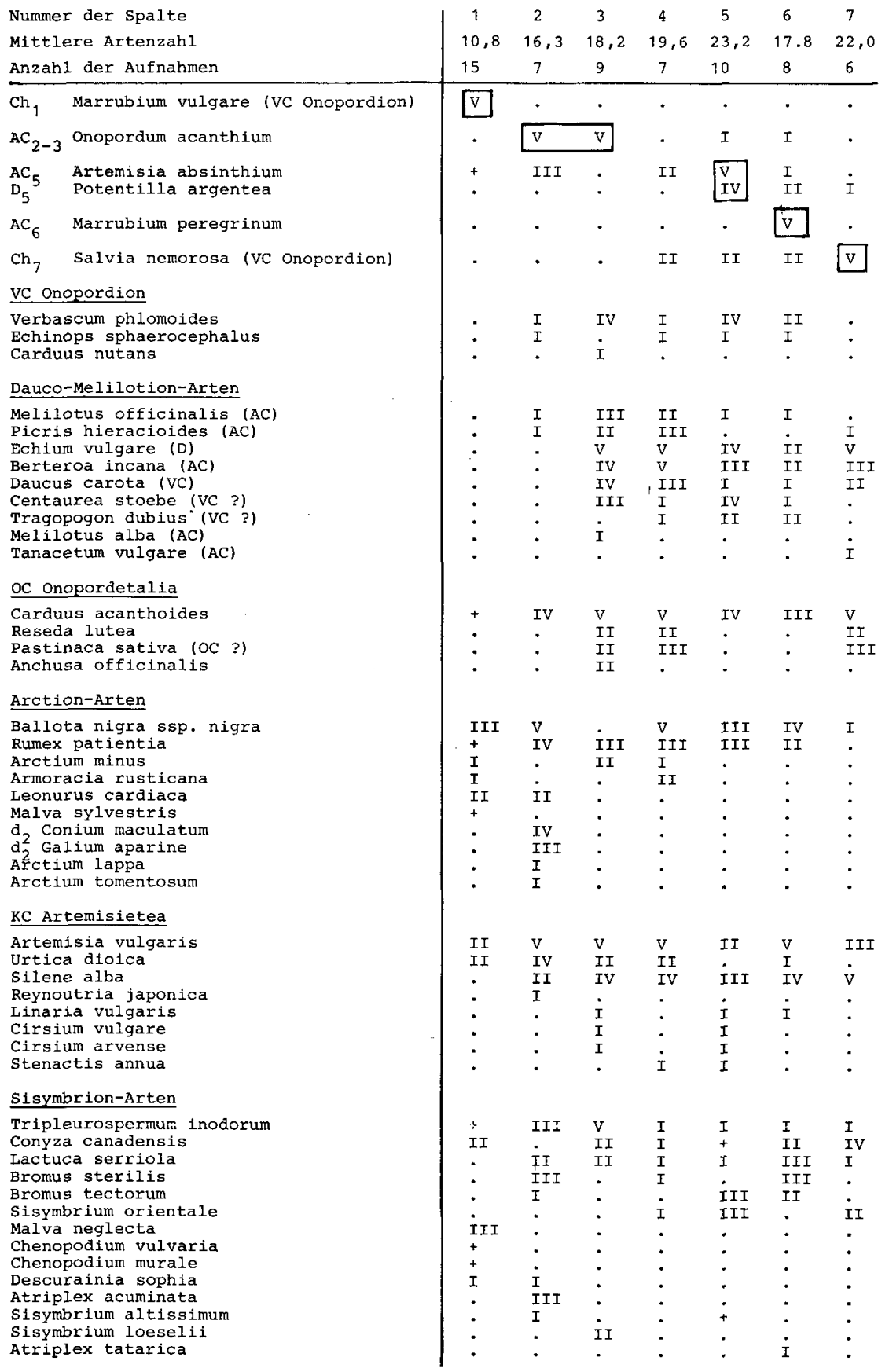


Tabelle 15. (Fortsetzung)

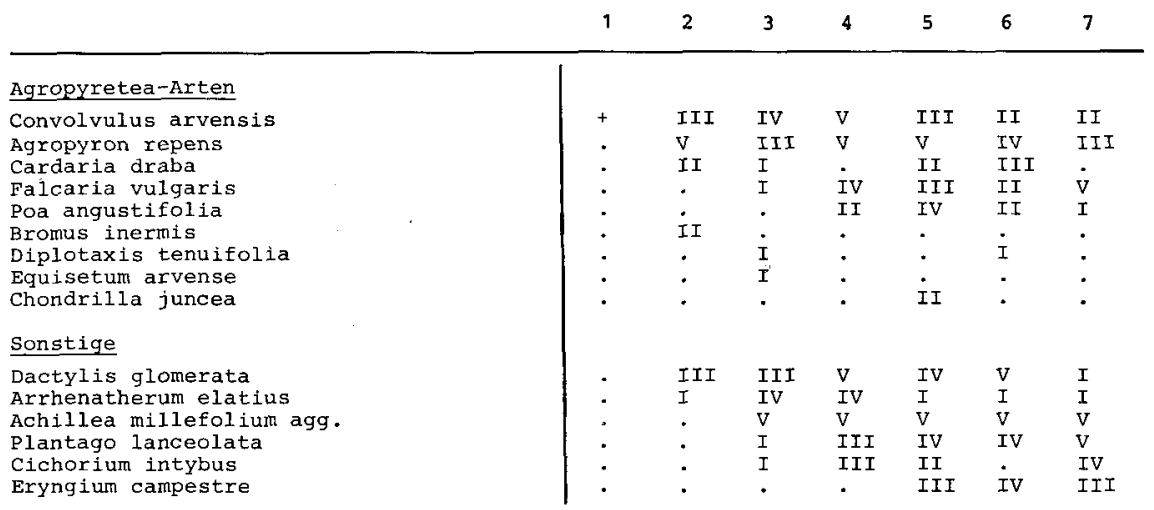

(zahlreiche Begleiter mit geringer stetigkeit)

Spalte 1: Marmubium vulgare-Gesellschaft

Spalte 2: Onopordetum acanthii Br.-Bl. (1923 n.n.) 1926 arctietosum

Spalte 3: Onopordetum acanthii Br.-Bl. (1923 n.n.) 1926 echietosum

Spalte 4: Basalgesellschaft Carduus acantboides-[Onopordetalia]

Spalte 5: Potentillo-Artemisietum absinthii Faliński 1965

Spalte 6: Salvio-Marrubietum peregrini Mucina 1981

Spalte 7: Salvia nemorosa-Straßenrandgesellschaft

\subsection{Klettenfluren (Arction lappae Tx. $1937 \mathrm{em} .1950$ )}

4.4.1. Arctio-Artemisietum (Tx. 1942) Oberd. ap. Oberd. et al. 1967, Kletten-Beifuß-Gestrüpp (Tab, 16)

Kletten-Beifuß-Gestrüppe stellen die wichtigste A r c t i o n-Gesellschaft der Seewinkel-Dörfer dar. Sie werden von Arctium lappa, Artemisia vulgaris, Ballota nigra und Urtica dioica aufgebaut. Fast immer findet sich auch Sambucus nigra, woraus auf die Sukzession zu ruderalen Gebüschen geschlossen werden kann.

\subsubsection{Conietum maculati Pop (1965) 1968 (Tab. 16)}

An den Dorfrändern fallen mannshohe Schierlingsgestrüppe auf, die mitunter hunderte von Quadratmetern bedecken können. Entsprechend ihrer Artenzusammensetzung gehören sie zweifellos zum A r c t i o n, lassen sich aber nicht mehr $\operatorname{dem} \mathrm{Arct}$ io-A r t e $\mathrm{m}$ i s e $\mathrm{t} \mathrm{u} \mathrm{m}$ anschließen. Sie bilden vielmehr den feuchtigkeitsbedürftigen Flügel des $\mathrm{A} \mathrm{r} \mathrm{c}$ i o $\mathrm{n}$. Da Lamium album fehlt, lassen sich diese Bestände nur schwer dem L a m i o - C o n i e t u m Oberd. 1957 zuordnen, was z.B. auch für die Conium maculatum-Fluren der Südalpen gilt (BRANDES \& BRANDES 1981, BRANDES 1987). Wir folgen daher HRUŠKA (1982), die die mittelitalienischen Conium maculatum-Bestände dem C o n i e $\mathrm{t} \mathrm{u} \mathrm{m} \mathrm{m}$ a c u l a t i Pop (1965) 1968 anschließt. 
Tabelle 16. Arctio-Artemisietum (Tx. 1942) Oberd. ap. Oberd. et al. 1967 und Conietum maculati Pop (1965) 1968.

\begin{tabular}{|c|c|c|c|c|c|c|c|c|}
\hline Nummer der Aufnahme & 1 & 2 & 3 & 4 & 5 & 6 & 7 & 8 \\
\hline Fläche $\left(m^{2}\right)$ & 6 & 30 & 10 & 10 & 70 & 80 & 30 & 100 \\
\hline Vegetationsbedeckung $(z)$ & 95 & 100 & 100 & 90 & 100 & 100 & 100 & 100 \\
\hline Artenzahl & 15 & 12 & 12 & 13 & 16 & 14 & 16 & 22 \\
\hline \multicolumn{9}{|l|}{ AC Arctio-Artemisietum } \\
\hline $\begin{array}{l}\text { Artemisia vulgaris } \\
\text { Arctium lappa }\end{array}$ & $\begin{array}{l}2.2 \\
2.2 \\
\end{array}$ & $\begin{array}{l}2.1 \\
2.2 \\
\end{array}$ & $\begin{array}{l}3.4 \\
3.3 \\
\end{array}$ & $\begin{array}{l}2.3 \\
2.2 \\
\end{array}$ & $\begin{array}{l}2.2 \\
2.2 \\
\end{array}$ & $\begin{array}{l}1.1 \\
1.1 \\
\end{array}$ & $\begin{array}{c}2.2 \\
\cdot\end{array}$ & $\begin{array}{c}2.2 \\
\cdot\end{array}$ \\
\hline \multicolumn{9}{|l|}{ AC Conietum maculati } \\
\hline $\begin{array}{l}\text { Conium maculatum } \\
\text { d Rumex patientia } \\
\text { d Galium aparine }\end{array}$ & $\dot{.}$ & $\dot{.}$ & $\begin{array}{r}\cdot \\
+.2\end{array}$ & $\dot{ن}$ & $\begin{array}{c}1.2 \\
:\end{array}$ & \begin{tabular}{|l}
4.4 \\
1.2 \\
2.2 \\
\end{tabular} & $\begin{array}{l}3.2 \\
1.2 \\
2.2 \\
\end{array}$ & $\begin{array}{l}4 \cdot 3 \\
1.1 \\
2.2 \\
\end{array}$ \\
\hline \multicolumn{9}{|l|}{ VC Arction } \\
\hline $\begin{array}{l}\text { Ballota nigra ssp. nigra } \\
\text { Arctium minus } \\
\text { Leonurus cardiaca }\end{array}$ & $\begin{aligned} 2.2 \\
2.2 \\
.\end{aligned}$ & $\begin{array}{c}1.2 \\
:\end{array}$ & $\begin{array}{c}2.2 \\
:\end{array}$ & $\begin{array}{l}2.3 \\
1.1 \\
.\end{array}$ & $\begin{array}{c}1.2 \\
:\end{array}$ & $\begin{array}{l}1.2 \\
:\end{array}$ & $\begin{array}{l}3.3 \\
2.2 \\
.\end{array}$ & $\begin{array}{l}2.3 \\
1.1 \\
1.2\end{array}$ \\
\hline \multicolumn{9}{|l|}{$\begin{array}{l}\text { Artemisietea- und Galio- } \\
\text { Calystegietalia-Arten }\end{array}$} \\
\hline Urtica dioica & 3.4 & 4.4 & 2.3 & 3.3 & 4.4 & 3.2 & 2.2 & . \\
\hline $\begin{array}{l}\text { Carduus crispus } \\
\text { Silene alba }\end{array}$ & $\cdot$ & 2.3 & $\cdot$ & · & $\begin{array}{l}3.2 \\
1.2\end{array}$ & $\cdot$ & $\cdot$ & $\cdot$ \\
\hline Cuscuta europaea & : & + & . & : & 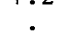 & $\dot{.}$ & : & : \\
\hline Calystegia sepium & - & . & 1.1 & . & . & - & - & - \\
\hline Cirsium arvense & - & - & . & + & $\cdot$ & - & - & - \\
\hline Lamium maculatum & . & - & - & - & 1.2 & $\cdot$ & - & . \\
\hline Alliaria petiolata & . & . & . & . & . & +.2 & . & \\
\hline Stenactis annua & - & - & - & - & . & • & . & 1.2 \\
\hline Geum urbanum & - & $\cdot$ & $\cdot$ & • & . & - & - & + \\
\hline \multicolumn{9}{|l|}{ Begleiter } \\
\hline Sambucus nigra & 1.1 & 1.1 & . & 1.1 & + & 1.1 & 1.1 & . \\
\hline Agropyron repens & $\cdot$ & 2.3 & $\cdot$ & +.2 & 2.3 & 3.3 & $\cdot$ & $\cdot$ \\
\hline Achiliea millefolium agg. & - & • & 1.2 & $\cdot$ & 1.2 & +.2 & $\cdot$ & 2.2 \\
\hline Convolvulus arvensis & . & . & • & 1.2 & 1.2 & . & 1.2 & 1.2 \\
\hline Dactylis glomerata & 1.1 & . & . & 2.2 & $\cdot$ & - & $\cdot$ & 2.2 \\
\hline Atriplex patula & 1.2 & . & 1.2 & . & . & . & 1.2 & . \\
\hline Hordeum murinum & 1.2 & . & . & +.2 & . & . & 1.2 & . \\
\hline Bromus sterilis & 1.2 & . & . & . & . & . & 2.2 & 2.2 \\
\hline Lolium perenne & 1.2 & . & . & 1.2 & . & . & . & . \\
\hline Taraxacum officinale & + & . & . & + & - & . & . & . \\
\hline Robinia pseudacacia juv. & 1.1 & . & + & . & $\cdot$ & - & - & - \\
\hline Heracleum sphondylium & . & 1.1 & . & . & 1.1 & . & . & . \\
\hline Anthriscus sylvestris & . & 1.1 & . & . & 1.2 & . & . & . \\
\hline Bromus inermis & . & 1.2 & . & . & 1.2 & . & . & . \\
\hline Lactuca serriola & . & . & + & . & . & . & + & + \\
\hline Conyza canadensis & . & . & 1.2 & . & . & . & . & 2.3 \\
\hline Arrhenatherum elatius & - & . & $\cdot$ & - & 1.2 & - & - & + \\
\hline Onopordum acanthium & . & . & . & . & . & + & . & + \\
\hline
\end{tabular}

Außerdem in $\mathrm{Nr}$. 1: 1.2 Plantago major, 1.2 Cichorium intybus. Nr. $3: 2.2$ Lycium barbarum. Nr. 6: 2.2 Carduus acanthoides, 1.2 Galium mollugo agg. Nr. 7: 1.1 Pastinaca sativa, + Taraxacum officinale, r Plantago lanceolata. Nr. 8: 2.2 Artemisia absinthium, 2.2 Poa pratensis, 1.2 Reseda lutea, 1.2 Bromus hordeaceus ssp. hordeaceus, + Tragopogon dubius. 


\subsubsection{Sambucetum ebuli Felf. 1942}

Vor allem an Ortsrändern, Straßenböschungen und Feldwegrändern finden sich mitunter große Sambucus ebulus-Bestände. Das Areal dieser konkurrenzkräftigen Staude umfaßt Süd- und Südosteuropa, große Teile West-, Mittel- und Osteuropas, das nördliche Atlasgebiet, Randbereiche Kleinasiens sowie Teile von Iran und Kaschmir. Sambucus ebulus ist Kennart mindestens zweier Assoziationen: in Südost(mittel)europa ist das $\mathrm{S}$ a $\mathrm{m}$ b u c e t u m e b u l i Felf. 1942 vertreten, während im (sub)atlantisch getönten Mittel- und Westeuropa das $\mathrm{He} \mathrm{ra} \mathrm{cl} \mathrm{e} \mathrm{o-}$ $\mathrm{S}$ a m bu cet u m e buli Brandes 1985 gedeiht.

Mit diversen A r c t i n - und On o pordet a li a-Arten gehören die Bestände im Untersuchungsgebiet wohl bereits zum $\mathrm{S}$ a $\mathrm{mbu} \mathrm{c}$ e $\mathrm{tu} \mathrm{m}$ eb u li Felf. 1942 (vgl. BRANDES 1982a).

Einzelaufnahme 9:

Pamhagen. $50 \mathrm{~m}^{2}$, D $98 \%$. 15.8.1985:

AC: 5.5 Sambucus ebulus; DA: +.2 Hordeum murinum, + Rumex patientia, + Silene alba; KC: 1.2 Galium aparine, 3.3 Urtica dioica, 1.2 Artemisia vulgaris; B: 1.2 Agropyron repens, 1.2 Dactylis glomerata, 1.2 Bromus sterilis, + Convolvulus arvensis.

\subsection{Nitrophile Säume (Glechometalia hederaceae Tx. in Tx. et Brun-Hool 1975)}

Nitrophile Säume sind in den Dörfern des Seewinkels selten, insbesondere scheint das U r t i co-A e g o p o d i e $\mathrm{t} \mathrm{m}$ und mit ihm der ganze Unteryerband $\mathrm{L}$ a $\mathrm{m}$ i o-A e g o p od i e $\mathrm{n}$ i o $\mathrm{n}$ völlig zu fehlen oder zumindest sehr selten $\mathrm{zu}$ sein.

Alte Parkanlagen wie die Schloßparks von Kittsee und von Eisenstadt beherbergen mit dem $\mathrm{Ch}$ elidon io-Parietariet um officinalis eine schwach thermophile Saumgesellschaft, die aus den myrmekochoren Arten Parietaria officinalis, Chelidonium majus, Viola odorata und Glecboma bederacea aufgebaut wird. Im Burgenland findet sich vor allem die Subassoziation von Ballota nigra. Aufnahmen aus dem nördlichen Burgenland sind in Tabelle 2 bei BRANDES (1985 b) zusammengestellt.

Im Schloßpark von Halbturn wurden Ballota nigra-Säume mit den folgenden Arten notiert: Alliaria petiolata, Ballota nigra ssp. nigra, Bracbypodium sylvaticum, Bryonia dioica, Chelidonium majus, Clematis vitalba, Geranium robertianum, Geum urbanum, Glechoma bederacea, Lapsana communis, Sambucus nigra, Urtica dioica und Viola odorata. Im Eisenstädter Schloßpark ist ebenso wie in den Leitha-Auen Geranium sibiricum in nitrophilen Säumen eingebürgert.

Die meisten nitrophilen Säume lassen sich keiner Assoziation zuordnen, sondern sind als Basal-bzw. Fragmentgesellschaften anzusehen.

Einzelaufnahme 10:

Nordexponierter Mauerfuß in Deutsch-Jahrndorf, zu ca. $80 \%$ von Robinien beschattet. $8 \mathrm{~m}^{2}$, D $100 \% .13 .8 .1985$ :

4.4 Glechoma bederacea, 1.2 Cruciata laevipes, 1.2 Geranium robertianum,

+ Geum urbanum, + Ballota nigra, + Cirsium vulgare; B: 2.2 Sambucus 
nigra juv., 2.2 Ranunculus repens, 1.2 Dactylis glomerata, 1.2 Festuca rubra agg., 1.1 Lolium perenne, + Poa annua, + Acbillea millefolium agg., +Polygonum bydropiper, + Soncbus oleraceus, $\mathrm{r}$ Malva neglecta .

In Entwässerungsgräben entlang der Bahnstrecke Neusiedl-Pamhagen gedeihen Rubus caesius-Bestände in unmittelbarer Nähe zur Panicum capillare-Gesellschaft.

Einzelaufnahme 11:

Frauenkirchen, $16 \mathrm{~m}^{2}$. D $100 \%, 13.8 .1985$ :

OC-KC: 4.4 Rubus caesius, 2.2 Calystegia sepium, 2.2 Urtica dioica, 2.2

Galium aparine, 2.2 Ballota nigra, +.2 Artemisia vulgaris, + Reseda lutea;

B: 2.2 Agropyron repens, 1.2 Convolvulus arvensis, 1.2 Lactuca serriola.

Das Vorkommen von Antbriscus cerefolium läßt schließlich das A $\mathrm{n} t \mathrm{~h} \mathrm{r}$ is c e t u m c e r efoli i-t r i c hos perm a e Hejný et Krippelová in HEjNÝ et al. 1979 erwarten; leider liegen bislang keine Aufnahmen solcher Bestände vor.

\subsection{Ruderale Gebüsche}

Die ruderalen Gebüsche werden hauptsächlich von Neophyten aufgebaut, von den einheimischen Gehölzen ist lediglich Sambucus nigra häufiger vertreten.

\subsubsection{Lycium barbarum-Bestände (Tab. 17)}

Lycium barbarum-Bestände gehören zur typischen Dorfvegetation des Untersuchungsgebietes; man findet sie vor allem entlang von Zäunen sowie in den Kellergassen. Neben Lycium barbarum erreichen nur Ballota nigra und Agropyron repens höhere Stetigkeit. Diese Bocksdorn-Gestrüppe entsprechen in ihrer Artenzusammensetzung zwar dem $\mathrm{L} \mathrm{y} \mathrm{c} \mathrm{i} \mathrm{e} \mathrm{t} \mathrm{u} \mathrm{h}$ a $\mathrm{li} \mathrm{m}$ if ol ia e Felf. 1942, sollten aber trotzdem nicht als eine eigene Assoziation angesehen werden, da es sich vielmehr um (kaum trennbare) lineare Komplexe aus Sträuchern und zugehörigen ruderalen Säumen handelt.

\subsubsection{Ailanthus altissima-Robinia pseudacacia-Gehölze}

Ailantbus altissima- und Robinia pseudacacia-Bestände geben den Scheunengassen ihr charakteristisches Gepräge. Beide Gehölzarten besitzen im Seewinkel eine erstaunliche Konkurrenzkraft.

Einzelaufnahme 12:

Deutsch Jahrndorf. $100 \mathrm{~m}^{2}$. 13.8.1985:

Baumschicht: 3.2 Robinia pseudacacia, 3.2 Ailantbus altissima;

Strauchschicht: 2.2 Sambucus nigra, 2.1 Robinia pseudacacia;

Krautschicht: 3.3 Ailantbus altissima juv., 3.3 Ballota nigra, 2.3 Hordeum murinum, 2.2 Leonurus cardiaca, 2.2 Cbenopodium album, 2.2 Bromus sterilis, 1.2 Urtica dioica, 1.1 Robinia pseudacacia juv., +.2 Artemisia vulgaris, + Chenopodium bybridum,+ Geum urbanum, + Acbillea millefolium agg.,$+\mathrm{Ta}^{-}$ raxacum officinale agg. 
Tabelle 17. Lycium barbarum-Bestände.

\begin{tabular}{|c|c|c|c|c|c|c|}
\hline Nummer der Aufnahme & 1 & 2 & 3 & 4 & 5 & 6 \\
\hline Fläche $\left(\mathrm{m}^{2}\right)$ & 20 & 15 & 15 & 6 & 15 & 10 \\
\hline Vegetationsbedeckung ( 8 ) & 100 & 100 & 98 & 95 & 100 & 98 \\
\hline Artenzah 1 & 11 & 12 & 14 & 12 & 10 & 13 \\
\hline Lycium barbarum & $4 \cdot 3$ & $4 \cdot 3$ & 4.4 & 3.3 & 3.2 & 3.2 \\
\hline $\begin{array}{l}\text { Ballota nigra ssp. nigra } \\
\text { Artemisia vulgaris }\end{array}$ & $\begin{array}{l}2.3 \\
1.2\end{array}$ & $\begin{array}{l}2.2 \\
2.2\end{array}$ & $\begin{array}{l}2.2 \\
2.2\end{array}$ & $\begin{array}{l}3.3 \\
2 \cdot 2\end{array}$ & $\begin{array}{l}3.3 \\
1.2\end{array}$ & 1.2 \\
\hline Urtica diolca & 3.3 & 2.2 & 2.2 & $\cdot$ & $\cdot$ & - \\
\hline Galium aparine & 1.2 & 2.2 & 1.2 & - & $\cdot$ & - \\
\hline Silene alba & 1.2 & $\cdot$ & $\cdot$ & - & 1.2 & + \\
\hline Carduus acanthoides & • & - & 1.2 & - & • & - \\
\hline Reseda lutea & - & - & $\cdot$ & +.2 & $\therefore$ & - \\
\hline Bryonia alba & • & • & - & • & 2.2 & $\cdot$ \\
\hline Echinops sphaerocephalus & - & - & . & . & - & 2.2 \\
\hline Agropyron repens & 2.2 & 1.2 & 1.2 & 3.3 & 2.3 & • \\
\hline Convolvulus arvensis & 2.2 & $\cdot$ & 1.2 & $\cdot$ & $\cdot$ & 1.2 \\
\hline Conyza canadensis & 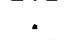 & 1.1 & + & + & - & $\cdot$ \\
\hline Galium mollugo agg. & 1.2 & . & +.2 & . & . & . \\
\hline Sambucus nigra & + & . & - & . & 2.1 & - \\
\hline Arrhenatherum elatius & 1.2 & - & - & - & - & 3.2 \\
\hline Chenopodium album & - & 1.1 & - & . & - & $\cdot$ \\
\hline Robinia pseudacacia juv. & - & 2.2 & - & - & - & - \\
\hline Amaranthus retroflexus & - & 1.2 & - & - & - & - \\
\hline Descurainia sophia & - & 1.1 & • & - & - & * \\
\hline Lactuca serriola & - & 2.2 & 1.2 & . & - & - \\
\hline Achillea millefolium agg. & - & . & 1.2 & - & - & - \\
\hline Phleum pratense & - & - & 1.2 & - & . & . \\
\hline Cichorium intybus & . & . & + & . & - & . \\
\hline Bromus sterilis & - & . & . & 1.2 & - & 1.2 \\
\hline Mercurialis annua & - & - & - & $r$ & - & 1.2 \\
\hline Hordeum murinum & • & . & . & 1.2 & - & • \\
\hline Setaria verticillata & - & . & - & +.2 & - & * \\
\hline Falcaria vulgaris & • & • & - & +.2 & - & - \\
\hline Fallopia convolvulus & . & . & . & + & • & * \\
\hline Digitaria sanguinalis & - & • & - & - & 1.2 & - \\
\hline Atriplex patula & * & . & - & - & + & - \\
\hline Sisymbrium orientale & $\cdot$ & - & - & - & + & - \\
\hline Medicago x varia & 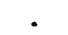 & - & . & - & - & 1.2 \\
\hline Delphinium consolida & - & . & . & - & - & 1.2 \\
\hline Keimlinge indet. & . & • & * & - & - & 1.2 \\
\hline Ailanthus altissima juv. & - & - & . & - & - & + \\
\hline Cannabis sativa & - & - & - & - & - & + \\
\hline
\end{tabular}

\section{Anmerkung:}

Die meisten der Ruderalgesellschaften wurden bereits von FORSTNER (1983, 1984) in sehr komprimierter Form für das östliche Österreich angegeben. Aus den Stetigkeitstabellen sind nur in begrenztem Maße Rückschlüsse auf die Dorfvegetation des Untersuchungsgebietes möglich. 
Zusarnmenfassung. Ziel der vorliegenden Arbeit war es, Flora und Vegetation der Dörfer des nordöstlichen Burgenlandes (Österreich) zu untersuchen und zu beschreiben. Auf der Grundlage einer floristischen Kartierung von 14 Dörfern sind Aussagen über die Häufigkeit dorftypischer Pflanzen möglich. Die hohe Stetigkeit vieler Arten ist charakteristisch; 88 Sippen wurden in mindestens der Hälfte der Ortschaften notiert. Die Dorfflora des Untersuchungsgebietes wird durch zahlreiche kontinentale und/oder thermophile Arten gekennzeichnet, wobei der Neophytenanteil recht hoch ist.

Der Vergleich zu Dörfern anderer Naturräume zeigt, daß es - auf ganz Mitteleuropa bezogen - nur wenige, ,typische Dorfpflanzen“"gibt. Die dörfliche Flora ist vielmehr von Landschaft zu Landschaft verschieden, vor allem aus klimatischen, aber auch aus edaphischen und kulturhistorischen Gründen.

Im Randbereich der burgenländischen Dörfer konnten sich Ruderalfluren in einem für Mitteleuropa ungewöhnlichen Ausmaße entwickeln. 25 Ruderalgesellschaften wurden mit Vegetationsaufnahmen belegt. Für das Gebiet charakteristische Pflanzengesellschaften sind A t r p li c e t u $\mathrm{m}$ a $\mathrm{t}$ a $\mathrm{r}$ i a e, Bg. Carduus acantboides-[O n o p or d e t a li a], Coniet $\mathrm{um}$ ma culati, Malvet um pusilla e, Panicum capillare-Gesellschaft, Onopordetum acanthii, Salvio-Marrubiet um peregrini,

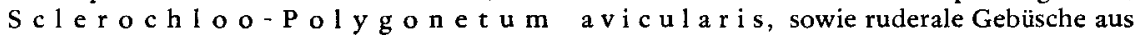
Lycium barbarum, Sambucus nigra, Ailantbus altissima und Robinia pseudacacia.

\section{Literatur}

Brandes, D. (1982): Das Atriplicetum nitentis Knapp 1945 in Mitteleuropa, insbesondere in Südost-Niedersachsen. - Doc. Phytosoc. N.S. 6: 131-153, Camerino.

- (1982a): Das Sambucetum ebuli im südlichen Mitteleuropa und seine geographische Gliederung. - Tuexenia 2: 47-60, Göttingen.

- (1985): Zur Verbreitung und Soziologie von Salvia nemorosa L. in Mitteleuropa. - Gött. Flor. Rundbr. 19: 29-34, Göttingen.

- (1985a): Die Ruderalvegetation im östlichen Niedersachsen: Syntaxonomische Gliederung, Verbreitung und Lebensbẹdingungen. - Habil.schrift TU Braunschweig. VI, 292 S., Braunschweig (Mskr.).

- (1985b): Nitrophile Saumgesellschaften in alten Parkanlagen und ihre Bedeutung für den Naturschutz. - Phytocoenologia 13: 451-462, Stuttgart, Braunschweig.

- (1987): Ruderal- und Saumgesellschaften des Südalpenrandes. - Tuexenia 7: 121-138, Göttingen.

Brandes, D. \& Brandes, E. (1981): Ruderal- und Saumgesellschaften des Etschtals zwischen Bozen und Rovereto, - Tuexenia 1: 99-138, Göttingen.

Dechent, H.-J., Müller-Schönborn, J. \& Sperber, H. (1986): Untersuchungen und Vorschläge zur Kartierung und Erhaltung der Dorfflora in Rheinland-Pfaiz. - Natur u. Landschaft 61: 268-274. Stuttgart.

Eichstädt, U. (1986): Die Verbreitung von Dorfunkräutern in einigen Dörfern im Ostteil des Kreises Pasewalk. - Bot. Rundbr. Bez. Neubrandenburg 18: 75-79, Neubrandenburg.

Eliáš, P. (1981): A thermo-xerophilous anthropogenic community: Marrubio peregrini-Salvietum nemorosae Eliáš 1980. - Feddes Repert. 92: 563-568, Berlin.

Faliński, J.B. (1971): Flora i roślinność synantropijna wsi i miast - próba analyizy porównawczej. - Mater. Zakl. Fitosoc. Stos. U.W. Warszawa-Białowieża 27: 15-37, Warszawa.

Feráková, V. \& Jarolímek, I. (1986): Anthropogenic changes in flora and vegetation of Bratislava. - In: Schubert, R. \& Hilbig, W. (Hrsg.): Erfassung und Bewertung anthropogener Vegetationsveränderungen. T. 1. - Wiss. Beitr. Martin-Luther-Univ. Halle-Wittenberg 1987/4 (P 26): 145-157, Halle.

Forstner, W. (1983, 1984): Ruderale Vegetation in Ost-Österreich. - Wiss. Mitt. Niederösterr. Landesmuseum 2: 19-133, 3: 11-91, Wien.

Hegi, G. (1975): Illustrierte Flora von Mitteleuropa. Bd. V, T. 4, Nachdr. d. 2. Aufl. - Berlin, Hamburg. 
Hilbrich, S., Kintzel, W. \& Lembcke, K. (1983): Zur Verbreitung einiger Dorfstraßenpflanzen im Kreis Lübz. - Bot. Rundbr. Bez. Neubrandenburg 14: 77-82, Neubrandenburg.

Hruška, K. (1982): La végétation sinanthropique de Camerino et des ses alentours. - In: Pedrotti, F.: Guide-Itinéraire de l'Excursion Internationale de Phytosociologie en Italie Centrale (2-11 juillet 1982). - S. 285-304, Camerino.

Janchen, E. (1977): Flora von Wien, Niederösterreich und Nordburgenland. 2. Aufl. 757 S., Wien.

Kletter, L. (1976): Klima, Wetter, Wasserhaushalt. - In: Naturgeschichte Osterreichs. 567 S., Wien.

Koch, K. (1958): Flora des Regierungsbezirks Osnabrück und der benachbarten Gebiete. 2. Aufl. - LV, 543 S., Osnabrück.

Kräftner, J. (1984): Österreichs Bauernhöfe. - 133 S., Innsbruck, Frankfurt a.M.

Krippelová, T. (1981): Synanthrope Vegetation des Beckens Kosická kotlina. - 214 S., Bratislava. (=Vegetácia CSSR, B 4).

Lienenbecker, H. (1986): Flora und Vegetation in den Dörfern des Kreises Lippe. - Lippische Mitt. a. Geschichte u. Landeskunde 55: 301-346, Detmold.

Mucina, L, (1981): Die Ruderal-Vegetation des nördlichen Teils der Donau-Tiefebene. 1. Onopordion acanthii-Verband. - Folia Geobot. Phytotax. 16: 225-263, Praha.

Niklfeld, H., Karrer, G., Gutermann, W. \& Schratt, L. (1986): Rote Liste gefährdeter Farnund Blütenpflanzen (Pteridophyta und Spermatophyta) Osterreichs. - In: Rote Listen gefährdeter Pflanzen Österreichs. - Grüne Reihe d. Bundesmin. f. Gesundheit u. Umweltschutz 5: 28-109, Wien.

Oberdorfer, E. (1983): Pflanzensoziologische Exkursionsflora. 5. Aufl. - 1051 S., Stuttgart.

- (Hrsg.) (1983a): Süddeutsche Pflanzengesellschaften. 2. Aufl. T. 3. - 455 S., Stuttgart.

Schaberg, F. (1983): Panicum capillare L. - Herkunft und Verbreitung einer bemerkenswerten Adventivpflanze unter besonderer Berücksichtigung der Fundorte in Sachsen. -- Gleditschia 10: 121-130, Berlin.

Stenzel, G. (1984): Das Dorf in Österreich. - $175 \mathrm{~S}$., Wien.

Sukopp, H. (1983): Die Bedeutung der Freilichtmuseen für den Arten- und Biotopschutz. Schriftenr. Stiftg. Schutze gefährdeter Pflanzen 3: 34-42, Bonn.

Wittig, R. \& Rückert, E. (1985): Die spontane Flora im Ortsbild nordrhein-westfälischer Dörfer. - Siedlg. u. Landsch. in Westfalen 17: 107-154, Münster.

Anschrift der Verfasser:

Uwe RAABE, Holtfeld 43, D-4807 Borgholzhausen.

Priv. Doz. Dr. Dietmar BRANDES, Universitätsbibliothek der Technischen Universität, Pockelsstraße 13, D-3300 Braunschweig. 